\title{
A SHARP SOBOLEV TRACE INEQUALITY INVOLVING THE MEAN CURVATURE ON RIEMANNIAN MANIFOLDS
}

\author{
TIANLING JIN AND JINGANG XIONG
}

Abstract. Let $(M, g)$ be a smooth compact $n$-dimensional Riemannian manifold with smooth boundary $\partial M$ for $n \geq 5$. We prove a trace inequality, that is

$$
\|u\|_{L^{q}(\partial M)}^{2} \leq S\left(\int_{M}\left|\nabla_{g} u\right|^{2} \mathrm{~d} v_{g}+\frac{n-2}{2} \int_{\partial M} h_{g} u^{2} \mathrm{~d} s_{g}\right)+A\|u\|_{L^{r}(\partial M)}^{2}
$$

for all $u \in H^{1}(M)$, where $S=\frac{2}{n-2} \omega_{n}^{-1 /(n-1)}$ with $\omega_{n}$ the volume of the unit sphere in $\mathbb{R}^{n}, q=\frac{2(n-1)}{n-2}, r=\frac{2(n-1)}{n}, h_{g}$ is the mean curvature of $\partial M, \mathrm{~d} v_{g}$ is the volume form of $(M, g), \mathrm{d} s_{g}$ is the induced volume form on $\partial M$, and $A$ is a positive constant depending only on $(M, g)$. This inequality is sharp in the sense that $S$ cannot be replaced by any smaller constant, $h$ in general cannot be replaced by any smooth function which is smaller than $h$ at some point on $\partial M$, and $r$ cannot be replaced by any smaller number.

\section{INTRODUCTION}

Recall that the sharp Sobolev trace inequality in the upper half space asserts

$$
\left(\int_{\partial \mathbb{R}_{+}^{n}}|u|^{\frac{2(n-1)}{n-2}} \mathrm{~d} x^{\prime}\right)^{\frac{n-2}{n-1}} \leq S \int_{\mathbb{R}_{+}^{n}}|\nabla u|^{2} \mathrm{~d} x \quad \forall u \in C_{c}^{1}\left(\mathbb{R}_{+}^{n} \cup \partial \mathbb{R}_{+}^{n}\right),
$$

where $S=\frac{2}{n-2} \omega_{n}^{-1 /(n-1)}, x=\left(x^{\prime}, x_{n}\right)$, and $\omega_{n}$ is the volume of the unit sphere in $\mathbb{R}^{n}, n \geq 3$. The best constant and the extremal functions of (1.1) were found by Escobar [14] and Beckner [5] independently. Indeed, as pointed out in [5], (1.1) follows from the sharp fractional Sobolev inequality, because

$$
\left(\int_{\mathbb{R}^{n-1}} v^{\frac{2(n-1)}{n-2}} \mathrm{~d} x^{\prime}\right)^{\frac{n-2}{n-1}} \leq S\|v\|_{\dot{H}^{1 / 2}\left(\mathbb{R}^{n-1}\right)}^{2}
$$

and

$$
\|v\|_{\dot{H}^{1 / 2}\left(\mathbb{R}^{n-1}\right)}^{2}=\int_{\mathbb{R}_{+}^{n}}|\nabla u|^{2} \mathrm{~d} x
$$

where $u$ is the harmonic extension of $v$ in the upper half space, and the equality of (1.2) holds if and only if $v\left(x^{\prime}\right)$ takes the form

$$
v\left(x^{\prime}\right)=c\left(\frac{\lambda}{1+\lambda^{2}\left|x^{\prime}-x_{0}^{\prime}\right|}\right)^{\frac{n-2}{2}}
$$

for some $c \in \mathbb{R}, \lambda>0$, and $x_{0}^{\prime} \in \mathbb{R}^{n-1}$, which is proved by Lieb [27].

Received by the editors December 30, 2013 and, in revised form, March 3, 2014.

2010 Mathematics Subject Classification. Primary 46 E35.

The second author was supported in part by the First Class Postdoctoral Science Foundation of China (No. 2012M520002). 
For any bounded smooth domain $\Omega \subset \mathbb{R}^{n}$, the sharp trace inequality

$$
\|u\|_{L^{\frac{2(n-1)}{n-2}(\partial \Omega)}}^{2} \leq S\|\nabla u\|_{L^{2}(\Omega)}^{2}+A(\Omega)\|u\|_{L^{2}(\partial \Omega)}^{2} \quad \forall u \in H^{1}(\Omega),
$$

where $A(\Omega)>0$ depends only on $\Omega$, was established by Adimurthi-Yadava [1] for $n \geq 5$ and by Li-Zhu [25] on Riemannian manifolds in all dimensions. The sharp Sobolev inequality

$$
\|u\|_{L^{\frac{2 n}{n-2}}(\Omega)}^{2} \leq 2^{\frac{2}{n}} S_{n}\|\nabla u\|_{L^{2}(\Omega)}^{2}+A(\Omega)\|u\|_{L^{2}(\partial \Omega)}^{2} \quad \forall u \in H^{1}(\Omega),
$$

where $S_{n}$ is the best Sobolev constant, was initially proved by Brezis-Lieb [6] when $\Omega$ is the unit ball, and later by Adimurthi-Yadava [1] for $n \geq 5$ and by Li-Zhu 26] on Riemannian manifolds in all dimensions. We also obtained similar sharp weighted trace inequalities in 21 and some remainder terms in sharp fractional Sobolev inequalities in [20].

In this and a forthcoming paper, we would like to examine the boundary $L^{2}$ term in (1.3) and (1.4) further. The main result of the present paper is as follows.

Theorem 1.1. Let $\Omega$ be a bounded smooth domain in $\mathbb{R}^{n}$ with $n \geq 5$. There exists a positive constant $A(\Omega)$ depending only on $\Omega$ such that for all $u \in H^{1}(\Omega)$,

$$
\left(\int_{\partial \Omega}|u|^{q} \mathrm{~d} s\right)^{2 / q} \leq S\left(\int_{\Omega}|\nabla u|^{2} \mathrm{~d} x+\frac{n-2}{2} \int_{\partial \Omega} h u^{2} \mathrm{~d} s\right)+A(\Omega)\|u\|_{L^{r}(\partial \Omega)}^{2},
$$

where $q=\frac{2(n-1)}{n-2}, r=q^{\prime}=\frac{2(n-1)}{n}$, and $h(x)$ is the mean curvature of $\partial \Omega$ at $x$.

The inequality (1.5) also holds on Riemannian manifolds with boundaries.

Theorem 1.2. Let $(M, g)$ be a smooth compact n-dimensional Riemannian manifold with smooth boundary $\partial M$ for $n \geq 5$. Then there exists a positive constant $A(M, g)$ depending only on $M, g$ such that for all $u \in H^{1}(M)$,

$$
\left(\int_{\partial M}|u|^{q} \mathrm{~d} s_{g}\right)^{2 / q} \leq S\left(\int_{M}\left|\nabla_{g} u\right|^{2} \mathrm{~d} v_{g}+\frac{n-2}{2} \int_{\partial M} h_{g} u^{2} \mathrm{~d} s_{g}\right)+A\|u\|_{L^{r}(\partial M)}^{2},
$$

where $h_{g}$ is the mean curvature of $\partial M$ with respect to the metric $g, \mathrm{~d} v_{g}$ is the volume form of $(M, g)$, and $\mathrm{d} s_{g}$ is the induced volume form on $\partial M$.

The inequality (1.6) is sharp in the following sense. It would fail if $S$ is replaced by any smaller constant. In general, $h$ cannot be replaced by any smooth function which is smaller than $h$ at some point on $\partial M$, and $r$ cannot be replaced by any smaller number.

The best constant $S$ has been demonstrated to be crucial in the study of the Yamabe problem on manifolds with boundaries; see, e.g., Escobar [13. The effect of mean curvatures in sharp Hardy-Sobolev inequalities with a singularity on the boundary has been studied by Ghoussoub-Robert [15]. In [21], we proved a sharp weighted Sobolev trace inequality on Riemannian manifolds which would fail if the mean curvature is positive somewhere. The effect of scalar curvatures for sharp Sobolev inequalities on compact manifolds has been studied by Li-Ricciardi 23], Hebey [18, and references therein.

The sharp Sobolev inequality (1.6) is in the same spirit of a conjecture posed by Aubin [2, which has been confirmed through the work of Hebey-Vaugon [19], Aubin-Li [3], and Druet [10, 11]; see also Druet-Hebey [12] and Hebey [17]. The 
procedure to prove those types of inequalities is by contradiction. A key point is to derive the asymptotical behavior of extremal functions near their energy concentration points. However, to establish (1.6) requires more precise estimates, in particular the error estimates between the extremal functions and some properly chosen bubbles.

\section{Preliminaries}

We prove inequality (1.6) by contradiction. Suppose that for every large $\alpha>1$, there exists $\tilde{u} \in H^{1}(M)$ such that

$$
\left(\int_{\partial M}|\tilde{u}|^{q} \mathrm{~d} s_{g}\right)^{2 / q}>S\left(\int_{M}\left|\nabla_{g} \tilde{u}\right|^{2} \mathrm{~d} v_{g}+\frac{n-2}{2} \int_{\partial M} h_{g} \tilde{u}^{2}\right)+\alpha\|\tilde{u}\|_{L^{r}(\partial M, g)}^{2} .
$$

Define

$I_{\alpha}(u)=\frac{\int_{M}\left|\nabla_{g} u\right|^{2} \mathrm{~d} v_{g}+\frac{n-2}{2} \int_{\partial M} h_{g} u^{2}+\alpha\|u\|_{L^{r}(\partial M, g)}^{2}}{\|u\|_{L^{q}(\partial M)}^{2}}, \quad \forall u \in H^{1}(M) \backslash H_{0}^{1}(M)$.

It follows from the contradiction hypothesis that for all large $\alpha$,

$$
\ell_{\alpha}:=\inf _{H^{1}(M) \backslash H_{0}^{1}(M)} I_{\alpha}<\frac{1}{S} .
$$

Even though the functional $I_{\alpha}$ involves a critical Sobolev exponent, the above strict inequality implies the existence of a minimizer, namely,

Proposition 2.1. For every $\alpha>0, \ell_{\alpha}$ is achieved by a nonnegative $u_{\alpha} \in H^{1}(M) \backslash$ $H_{0}^{1}(M)$ with

$$
\int_{\partial M} u_{\alpha}^{q} \mathrm{~d} s_{g}=1 .
$$

Consequently, $u_{\alpha} \in C^{\infty}(M) \cap C^{1, r-1}(\bar{M})$ satisfies the Euler-Lagrange equation

$$
\begin{cases}-\Delta_{g} u_{\alpha}=0 & \text { on } M, \\ \frac{\partial_{g} u_{\alpha}}{\partial \nu}+\frac{n-2}{2} h_{g} u_{\alpha}+\alpha\left\|u_{\alpha}\right\|_{L^{r}(\partial M)}^{2-r} u_{\alpha}^{r-1}=\ell_{\alpha} u_{\alpha}^{q-1} & \text { on } \partial M,\end{cases}
$$

where $\partial_{g} / \partial \nu$ denotes the differentiation in the direction of the unit outer normal of $\partial M$ with respect to $g$.

Proof. The existence of a minimizer follows from the standard subcritical exponent approximating method and Moser's iteration argument; see, e.g., Proposition 2.1 of 13. A calculus of variations argument shows that $u_{\alpha}$ is a weak solution of the Euler-Lagrange equation (2.2). Finally, $u_{\alpha} \in C^{\infty}(M) \cap C^{1, r-1}(\bar{M})$ follows from the regularity theory in 8 .

Lemma 2.1. For every $\varepsilon>0$, there exists a constant $B(\varepsilon)>0$ depending on $\varepsilon$ such that

$$
\left(\int_{\partial M}|u|^{q} \mathrm{~d} s_{g}\right)^{2 / q} \leq(S+\varepsilon) \int_{M}\left|\nabla_{g} u\right|^{2} \mathrm{~d} v_{g}+B(\varepsilon)\|u\|_{L^{r}(\partial M, g)}^{2},
$$

for all $u \in H^{1}(M)$. 
Proof. By compactness, we have that for every $\varepsilon>0$ there exists a positive constant $\tilde{B}(\varepsilon)$ such that

$$
\int_{\partial M} u^{2} \mathrm{~d} s_{g} \leq \varepsilon \int_{M}\left|\nabla_{g} u\right|^{2} \mathrm{~d} v_{g}+\tilde{B}(\varepsilon)\|u\|_{L^{r}(\partial M, g)}^{2} .
$$

Hence, the proposition follows from the sharp trace inequality in Theorem 0.1 in [25].

Note that

$$
\begin{aligned}
I_{\alpha}\left(u_{\alpha}\right) & =\int_{M}\left|\nabla_{g} u_{\alpha}\right|^{2} \mathrm{~d} v_{g}+\frac{n-2}{2} \int_{\partial M} h_{g} u_{\alpha}^{2}+\alpha\left\|u_{\alpha}\right\|_{L^{r}(\partial M, g)}^{2} \\
& \geq\left(1-\varepsilon \max _{\partial M}\left|h_{g}\right|\right) \int_{M}\left|\nabla_{g} u_{\alpha}\right|^{2} \mathrm{~d} v_{g}+(\alpha-B(\varepsilon))\left\|u_{\alpha}\right\|_{L^{r}(\partial M, g)}^{2} .
\end{aligned}
$$

This implies

$$
\int_{M}\left|\nabla_{g} u_{\alpha}\right|^{2} \mathrm{~d} v_{g} \leq \frac{2}{S}
$$

and

$$
\left\|u_{\alpha}\right\|_{L^{r}(\partial M, g)} \rightarrow 0 \quad \text { as } \alpha \rightarrow \infty \text {. }
$$

It follows that $u_{\alpha} \rightarrow \bar{u}$ in $H^{1}(M)$ for some $\bar{u} \in H_{0}^{1}(M)$. In fact, $\bar{u}=0$, a.e. on $M$. This is because $u_{\alpha}$ is harmonic on $M$ and thus it satisfies (see Proposition 2.1 in [16])

$$
\left\|u_{\alpha}\right\|_{L^{\frac{n r}{n-r}}(M, g)} \leq C(M, g, n)\left\|u_{\alpha}\right\|_{L^{r}(\partial M, g)},
$$

and $u_{\alpha} \rightarrow \bar{u}$ in $L^{\frac{n r}{n-r}}(M)$.

We claim that, as $\alpha \rightarrow \infty$,

$$
\ell_{\alpha} \rightarrow \frac{1}{S}
$$

and

$$
\alpha\left\|u_{\alpha}\right\|_{L^{r}(\partial M, g)}^{2} \rightarrow 0
$$

Indeed, by Lemma 2.1 and (2.3) for every $\varepsilon>0$

$$
\begin{aligned}
1 & \leq\left(S+\frac{\varepsilon}{2}\right) \int_{M}\left|\nabla_{g} u_{\alpha}\right|^{2} \mathrm{~d} v_{g}+B(\varepsilon)\left\|u_{\alpha}\right\|_{L^{r}(\partial M, g)}^{2} \\
& \leq(S+\varepsilon) \ell_{\alpha}+(2 B(\varepsilon)-\alpha S)\left\|u_{\alpha}\right\|_{L^{r}(\partial M, g)}^{2} .
\end{aligned}
$$

Thus

$$
\frac{1}{S+\varepsilon} \leq \ell_{\alpha}<\frac{1}{S}, \quad \frac{1}{2} \alpha S\left\|u_{\alpha}\right\|_{L^{r}(\partial M, g)}^{2} \leq(S+\varepsilon) \frac{1}{S}-1=\frac{\varepsilon}{S},
$$

if $\alpha S>4 B(\varepsilon)$. Hence, the claim follows.

By the maximum principle, there exists a point $x_{\alpha} \in \partial M$ such that

$$
u_{\alpha}\left(x_{\alpha}\right)=\max _{\bar{M}} u_{\alpha}>0
$$

In view of (2.5) and

$$
1=\int_{\partial M} u_{\alpha}^{q} \leq u_{\alpha}\left(x_{\alpha}\right)^{q-r} \int_{\partial M} u_{\alpha}^{r},
$$

we have $u_{\alpha}\left(x_{\alpha}\right) \rightarrow \infty$. Set $\mu_{\alpha}:=u_{\alpha}\left(x_{\alpha}\right)^{-2 /(n-2)}$. Then $\mu_{\alpha} \rightarrow 0$ as $\alpha \rightarrow \infty$.

Let $x=\left(x_{1}, \cdots, x_{n-1}, x_{n}\right)=\left(x^{\prime}, x_{n}\right)$ be the Fermi coordinate (see, e.g., 13]) at $x_{\alpha}$, where $\left(x_{1}, \cdots, x_{n-1}\right)$ are normal coordinates on $\partial M$ at $x_{\alpha}$ and $\gamma\left(x_{n}\right)$ is the 
geodesic leaving from $\left(x_{1}, \cdot, x_{n-1}\right)$ in the orthogonal direction to $\partial M$ and parameterized by arc length. In this coordinate system,

$$
\sum_{1 \leq i, j \leq n} g_{i j}(x) \mathrm{d} x_{i} \mathrm{~d} x_{j}=\mathrm{d} x_{n}^{2}+\sum_{1 \leq i, j \leq n-1} g_{i j}(x) \mathrm{d} x_{i} \mathrm{~d} x_{j} .
$$

Moreover, $g^{i j}$ has the following Taylor expansion near $\partial M$ :

Lemma 2.2 (Lemma 3.2 in [13]). For $\left\{x_{k}\right\}_{k=1, \cdots, n}$ small,

$$
\begin{aligned}
& g^{i j}(x)=\delta^{i j}+2 h^{i j}\left(x^{\prime}, 0\right) x_{n}+O\left(|x|^{2}\right), \\
& g^{i j} \Gamma_{i j}^{k}=O(|x|),
\end{aligned}
$$

where $i, j=1, \cdots, n-1$ and $h_{i j}$ is the second fundamental form of $\partial M$.

To proceed, we introduce some notation.

- For a domain $D \subset \mathbb{R}^{n}$ with boundary $\partial D$, we denote $\partial^{\prime} D$ as the interior of $\bar{D} \cap \partial \mathbb{R}_{+}^{n}$ in $\mathbb{R}^{n-1}=\partial \mathbb{R}_{+}^{n}$ and $\partial^{\prime \prime} D=\partial D \backslash \partial^{\prime} D$.

- For $\bar{x} \in \mathbb{R}^{n}, B_{r}(\bar{x}):=\left\{x \in \mathbb{R}^{n}:|x-\bar{x}|<r\right\}$ and $B_{r}^{+}(\bar{x}):=B_{r}(\bar{x}) \cap \mathbb{R}_{+}^{n}$, where $|\cdot|$ is the Euclidean distance. We will not keep writing the center $\bar{x}$ if $\bar{x}=0$.

For suitably small $\delta_{0}>0$ (independent of $\alpha$ ), we define $v_{\alpha}$ in a neighborhood of $x_{\alpha}=0$ by

$$
v_{\alpha}(y)=\mu_{\alpha}^{(n-2) / 2} u_{\alpha}\left(\mu_{\alpha} y\right), \quad x \in B_{\delta_{0} / \mu_{\alpha}}^{+},
$$

in the Fermi coordinate mentioned above. Then $v_{\alpha}$ satisfies

$$
\begin{cases}\Delta_{g_{\alpha}} v_{\alpha}=0, & \text { in } B_{\delta_{0} / \mu_{\alpha}}^{+}, \\ \frac{\partial_{g_{\alpha}} v_{\alpha}}{\partial \nu}+\frac{n-2}{2} h_{\alpha} v_{\alpha}+\epsilon_{\alpha} v_{\alpha}^{r-1}=\ell_{\alpha} v_{\alpha}^{q-1}, & \text { on } \partial^{\prime} B_{\delta_{0} / \mu_{\alpha}}^{+} \\ v_{\alpha}(0)=1, \quad 0 \leq v_{\alpha} \leq 1, & \end{cases}
$$

where $g_{\alpha}(x)=g_{i j}\left(\mu_{\alpha} x\right) \mathrm{d} x_{i} \mathrm{~d} x_{j}, h_{\alpha}$ is the mean curvature of $\partial^{\prime} B_{\delta_{0} / \mu_{\alpha}}$ with respect to the metric $g_{\alpha}$, and

$$
\epsilon_{\alpha}:=\alpha \mu_{\alpha}^{n-1-\frac{n-2}{2} r}\left\|u_{\alpha}\right\|_{L^{r}(\partial M)}^{2-r}
$$

Note that

$$
\epsilon_{\alpha}=\frac{\alpha\left\|u_{\alpha}\right\|_{L^{r}(\partial M)}^{2}}{\left(\max _{\bar{M}} u_{\alpha}\right)^{q-r} \int_{\partial M} u_{\alpha}^{r} \mathrm{~d} s_{g}} \leq \alpha\left\|u_{\alpha}\right\|_{L^{r}(\partial M)}^{2} \rightarrow 0 \quad \text { as } \alpha \rightarrow \infty,
$$

because $1=\int_{\partial M} u_{\alpha}^{q} \mathrm{~d} s_{g} \leq\left(\max _{\bar{M}} u_{\alpha}\right)^{q-r} \int_{\partial M} u_{\alpha}^{r} \mathrm{~d} s_{g}$. By the standard elliptic equations theory, for all $R>1$,

$$
\left\|v_{\alpha}\right\|_{C^{1, r-1}\left(\bar{B}_{R}^{+}\right)} \leq C(R) \text { for all sufficiently large } \alpha \text {. }
$$

Therefore, $v_{\alpha} \rightarrow U$ in $C_{l o c}^{1}\left(\overline{\mathbb{R}_{+}^{n}}\right)$ for some $U \in C_{l o c}^{1, r-1}\left(\overline{\mathbb{R}_{+}^{n}}\right) \cap C^{\infty}\left(\mathbb{R}_{+}^{n}\right)$ which satisfies

$$
\begin{cases}-\Delta U=0, & \text { in } \mathbb{R}_{+}^{n}, \\ -\partial_{y_{n}} U=\frac{1}{S} U^{q-1}, & \text { on } \partial \mathbb{R}_{+}^{n}, \\ U(0)=1, \quad 0 \leq U \leq 1 . & \end{cases}
$$

By the Liouville type theorem in [24,

$$
U\left(y^{\prime}, y_{n}\right)=\left(\frac{(n-2)^{2} S^{2}}{\left|y^{\prime}\right|^{2}+\left(y_{n}+(n-2) S\right)^{2}}\right)^{\frac{n-2}{2}}
$$


where $y^{\prime}=\left(y_{1}, \cdots, y_{n-1}\right)$. Denote $\lambda_{0}=(n-2) S$ and

$$
\begin{aligned}
U_{x_{0}, \lambda}\left(x^{\prime}, x_{n}\right) & :=\lambda^{-\frac{n-2}{2}} U\left(\left(x^{\prime}-x_{0}^{\prime}\right) / \lambda, x_{n} / \lambda\right) \\
& =\lambda_{0}^{\frac{n-2}{2}}\left(\frac{\lambda \lambda_{0}}{\left|x^{\prime}-x_{0}^{\prime}\right|^{2}+\left(x_{n}+\lambda \lambda_{0}\right)^{2}}\right)^{\frac{n-2}{2}},
\end{aligned}
$$

where $x_{0} \in \partial \mathbb{R}^{n}$ and $\lambda>0$. For brevity, we write $U_{\lambda}$ as $U_{0, \lambda}$. Hence, $U_{1}=U$.

Proposition 2.2. For every $\delta>0$,

$$
\lim _{\alpha \rightarrow \infty}\left(\int_{B_{\delta}^{+}}\left|\nabla_{g}\left(u_{\alpha}-U_{\mu_{\alpha}}\right)\right|^{2} \mathrm{~d} v_{g}+\int_{\partial^{\prime} B_{\delta}^{+}}\left|u_{\alpha}-U_{\mu_{\alpha}}\right|^{q} \mathrm{~d} s_{g}\right)=0 .
$$

Proof. We only prove that

$$
\lim _{\alpha \rightarrow \infty} \int_{B_{\delta}^{+}}\left|\nabla_{g}\left(u_{\alpha}-U_{\mu_{\alpha}}\right)\right|^{2} \mathrm{~d} v_{g}=0,
$$

and the other can be proved similarly.

For every given $\varepsilon>0$, one can find $\alpha_{0}>0$ such that for all $\alpha \geq \alpha_{0}$,

$$
\int_{M}\left|\nabla_{g} u_{\alpha}\right|^{2} \mathrm{~d} v_{g} \leq \frac{1}{S}+\varepsilon
$$

because $\lim _{\alpha \rightarrow \infty} \int_{M}\left|\nabla_{g} u_{\alpha}\right|^{2} \mathrm{~d} v_{g}=\frac{1}{S}$. Since $\int_{\mathbb{R}_{+}^{n}}|\nabla U|^{2}=\frac{1}{S}$, we can choose $R>0$ such that

$$
\int_{\mathbb{R}_{+}^{n} \backslash B_{R}^{+}}|\nabla U|^{2} \leq \varepsilon
$$

Note that

$$
\int_{B_{\delta / \mu_{\alpha}}^{+}}\left|\nabla_{g_{\alpha}} v_{\alpha}\right|^{2} \mathrm{~d} v_{g_{\alpha}}=\int_{B_{\delta}^{+}}\left|\nabla_{g} u_{\alpha}\right|^{2} \mathrm{~d} v_{g} \leq \frac{1}{S}+\varepsilon
$$

Hence

$$
\begin{aligned}
& \int_{B_{\delta}^{+}}\left|\nabla_{g}\left(u_{\alpha}-U_{\mu_{\alpha}}\right)\right|^{2} \mathrm{~d} v_{g} \\
& =\int_{B_{\delta / \mu_{\alpha}}^{+}}\left|\nabla_{g_{\alpha}}\left(v_{\alpha}-U\right)\right|^{2} \mathrm{~d} v_{g_{\alpha}} \\
& =\int_{B_{R}^{+}}\left|\nabla_{g_{\alpha}}\left(v_{\alpha}-U\right)\right|^{2} \mathrm{~d} v_{g_{\alpha}}+\int_{B_{\delta / \mu_{\alpha}}^{+} \backslash \bar{B}_{R}^{+}}\left|\nabla_{g_{\alpha}}\left(v_{\alpha}-U\right)\right|^{2} \mathrm{~d} v_{g_{\alpha}} \\
& \leq 2 \varepsilon+2 \int_{B_{\delta / \mu_{\alpha}}^{+} \backslash \bar{B}_{R}^{+}}\left|\nabla_{g_{\alpha}} v_{\alpha}\right|^{2} \mathrm{~d} v_{g_{\alpha}}+2 \int_{B_{\delta / \mu_{\alpha}}^{+} \backslash \bar{B}_{R}^{+}}\left|\nabla_{g_{\alpha}} U\right|^{2} \mathrm{~d} v_{g_{\alpha}} \\
& \leq 10 \varepsilon,
\end{aligned}
$$

where we used $\left\|v_{\alpha}-U\right\|_{C^{1}\left(\bar{B}_{R}^{+}\right)} \leq \varepsilon$ for large $\alpha$ and

$$
\begin{aligned}
& \int_{B_{\delta / \mu_{\alpha}}^{+} \backslash \bar{B}_{R}^{+}}\left|\nabla_{g_{\alpha}} v_{\alpha}\right|^{2} \mathrm{~d} v_{g_{\alpha}}+\int_{B_{\delta / \mu_{\alpha}}^{+} \backslash \bar{B}_{R}^{+}}\left|\nabla_{g_{\alpha}} U\right|^{2} \mathrm{~d} v_{g_{\alpha}} \\
& \quad \leq \frac{1}{S}+\varepsilon-\int_{B_{R}^{+}}\left|\nabla_{g_{\alpha}} v_{\alpha}\right|^{2} \mathrm{~d} v_{g_{\alpha}}+\frac{1}{S}+\varepsilon-\int_{B_{R}^{+}}\left|\nabla_{g_{\alpha}} U_{\alpha}\right|^{2} \mathrm{~d} v_{g_{\alpha}} \\
& \quad \leq 4 \varepsilon .
\end{aligned}
$$


Proposition 2.3. For all large $\alpha$,

$$
u_{\alpha}(x) \leq C \mu_{\alpha}^{\frac{n-2}{2}} \operatorname{dist}_{g}\left(x, x_{\alpha}\right)^{2-n} \quad \text { for all } x \in \bar{M},
$$

where $C>0$ depends only on $M, g$.

Let

$$
b_{\alpha}:= \begin{cases}\min \left\{\frac{n-2}{2} h_{g}+\alpha\left(\frac{\left\|u_{\alpha}\right\|_{L^{r}}}{u_{\alpha}}\right)^{2-r}, 1\right\}, & \text { if } u_{\alpha} \neq 0, \\ 1, & \text { if } u_{\alpha}=0 .\end{cases}
$$

To prove Proposition 2.3. we need the following lemma about Neumann functions.

Lemma 2.3. There exists a unique weak solution $G_{\alpha}$ of

$$
\begin{cases}-\Delta_{g} G_{\alpha}=0 & \text { on } M \\ \frac{\partial_{g} G_{\alpha}}{\partial \nu}+b_{\alpha} G_{\alpha}=\delta_{x_{\alpha}} & \text { on } \partial M\end{cases}
$$

satisfying $G_{a} \in W^{1, p}(M)$ for $1 \leq p<\frac{n}{n-1}$, where $\delta_{x_{\alpha}}$ is the delta measure centered at $x_{\alpha}$. Moreover, $G_{\alpha} \in C_{l o c}^{1}\left(\bar{M} \backslash\left\{x_{\alpha}\right\}\right)$ and

$$
C^{-1} \operatorname{dist}_{g}\left(x, x_{\alpha}\right)^{2-n} \leq G_{\alpha}(x) \leq C \operatorname{dist}_{g}\left(x, x_{\alpha}\right)^{2-n} \quad \text { for all } x \in \bar{M},
$$

where $C>0$ depends only on $M, g$.

Proof. We claim that

$$
\lim _{\alpha \rightarrow \infty} \operatorname{vol}_{g}\left\{b_{\alpha}<\frac{1}{2}\right\}=0 .
$$

Indeed, for every measurable set $E \subset \subset \partial M \cap\left\{u_{\alpha}>0\right\}$, we have

$$
\operatorname{vol}_{g}(E)=\int_{E} \mathrm{~d} s_{g}=\int_{E} u_{\alpha}^{r / 2} u_{\alpha}^{-r / 2} \mathrm{~d} s_{g} \leq\left\|u_{\alpha}\right\|_{L^{r}(E)}^{r / 2}\left\|u_{\alpha}^{-1}\right\|_{L^{r}(E)}^{r / 2} .
$$

It follows that

$$
\begin{aligned}
\left\|\left(\left\|u_{\alpha}\right\|_{L^{r}(\partial M)} u_{\alpha}^{-1}\right)^{2-r}\right\|_{L^{r /(2-r)}(E)} & =\left\|u_{\alpha}\right\|_{L^{r}(\partial M)}^{2-r}\left\|u_{\alpha}^{-1}\right\|_{L^{r}(E)}^{2-r} \\
& \geq\left(\operatorname{vol}_{g}(E)\right)^{2(2-r) / r} .
\end{aligned}
$$

Note that

$$
\alpha\left(\left\|u_{\alpha}\right\|_{L^{r}(\partial M)} u_{\alpha}^{-1}\right)^{2-r}<\frac{1}{2}\left(1+(n-2)\left|h_{g}\right|\right),
$$

if $b_{\alpha}<1 / 2$. Since $\left\{b_{\alpha}<\frac{1}{2}\right\} \subset \subset \partial M \cap\left\{u_{\alpha}>0\right\}$,

$$
\left(\operatorname{vol}_{g}\left\{b_{\alpha}<\frac{1}{2}\right\}\right)^{2(2-r) / r} \leq \frac{C}{\alpha} .
$$

We verified the claim. 
Notice that $b_{\alpha}$ is uniformly bounded and Lipschitz. Thus,

$$
\begin{aligned}
& \int_{M}\left|\nabla_{g} u\right|^{2} \mathrm{~d} v_{g}+\int_{\partial M} b_{\alpha} u^{2} \mathrm{~d} s_{g} \\
& \geq \int_{M}\left|\nabla_{g} u\right|^{2} \mathrm{~d} v_{g}+\frac{1}{2} \int_{\partial M} u^{2} \mathrm{~d} s_{g}-\int_{\partial M}\left(b_{\alpha}-\frac{1}{2}\right)^{-} u^{2} \mathrm{~d} s_{g} \\
& \geq \int_{M}\left|\nabla_{g} u\right|^{2} \mathrm{~d} v_{g}+\frac{1}{2} \int_{\partial M} u^{2} \mathrm{~d} s_{g}-\left\|\left(b_{\alpha}-\frac{1}{2}\right)^{-}\right\|_{L^{n-1}(\partial M)}\|u\|_{L^{q}(\partial M)}^{2} \\
& \geq \int_{M}\left|\nabla_{g} u\right|^{2} \mathrm{~d} v_{g}+\frac{1}{2} \int_{\partial M} u^{2} \mathrm{~d} s_{g} \\
& \quad-C\left\|\left(b_{\alpha}-\frac{1}{2}\right)^{-}\right\|_{L^{n-1}(\partial M)}\left(\int_{M}\left|\nabla_{g} u\right|^{2} \mathrm{~d} v_{g}+\int_{\partial M} u^{2} \mathrm{~d} s_{g}\right) \\
& \geq \frac{1}{4} \int_{M}\left|\nabla_{g} u\right|^{2} \mathrm{~d} v_{g}+\frac{1}{4} \int_{\partial M} u^{2} \mathrm{~d} s_{g}
\end{aligned}
$$

where we have used Theorem 0.1 in [25] and (2.15). Thus, it is coercive. Then it follows from standard elliptic theory (see, e.g., 7, 22 ) that there exists a unique solution of (2.13) in $W^{1, p}(M)$ for $1 \leq p<\frac{n}{n-1}$ satisfying (2.14). Note that the uniqueness is actually not needed in the rest of the paper.

Proof of Proposition 2.3. Set $\varphi_{\alpha}=\mu_{\alpha}^{(n-2) / 2} G_{\alpha}, w_{\alpha}=u_{\alpha} / \varphi_{\alpha}$, and $\hat{g}=\varphi_{\alpha}^{\frac{4}{n-2}} g$. By the conformal invariance (see, e.g., (1.8) in [13]), it is direct to verify that for $\alpha$ large, $w_{\alpha}$ satisfies

$$
\left\{\begin{aligned}
\Delta_{\hat{g}} w_{\alpha}= & 0 \text { on } M \\
\frac{\partial_{\hat{g}} w_{\alpha}}{\partial \nu}= & \ell_{\alpha} w_{\alpha}^{q-1} \\
& -w_{\alpha} \varphi_{\alpha}^{-\frac{n}{n-2}}\left(\frac{\partial_{g} \varphi_{\alpha}}{\partial \nu}+\frac{n-2}{2} h_{g} \varphi_{\alpha}+\alpha\left\|u_{\alpha}\right\|_{L^{r}}^{2-r} u_{\alpha}^{r-2} \varphi_{\alpha}\right) \quad \text { on } \partial M \backslash\left\{x_{\alpha}\right\} .
\end{aligned}\right.
$$

By our choice of $G_{\alpha}$, we have

$$
\begin{cases}\Delta_{\hat{g}} w_{\alpha}=0 & \text { on } M, \\ \frac{\partial_{\hat{g}} w_{\alpha}}{\partial \nu} \leq \ell_{\alpha} w_{\alpha}^{q-1} & \text { on } \partial M \backslash\left\{x_{\alpha}\right\} .\end{cases}
$$

Then the Moser iterations procedure on pp. 465-471 of [25] implies that

$$
\left\|w_{\alpha}\right\|_{L^{\infty}\left(M \backslash B_{\mu_{\alpha}}\left(x_{\alpha}\right)\right)} \leq C .
$$

Recall that $v_{\alpha} \rightarrow U$ in $C_{l o c}^{2}$, from which we also have $\left\|w_{\alpha}\right\|_{L^{\infty}\left(B_{\mu_{\alpha}}\left(x_{\alpha}\right)\right)} \leq C$. Proposition 2.3 follows immediately.

Corollary 2.1. For any small $\delta>0$, there exists a constant $C>0$ depending only on $M, g, \delta$ such that

$$
\int_{M \backslash B_{\delta / 2}\left(x_{\alpha}\right)}\left|\nabla_{g} u_{\alpha}\right|^{2} \leq C \mu_{\alpha}^{n-2},
$$

where $B_{\delta / 2}\left(x_{\alpha}\right)$ is centered at $x_{\alpha}$ with radius $\delta / 2$. Consequently, we can select $\delta_{\alpha} \in[\delta / 2, \delta]$ such that

$$
\int_{\partial B_{\delta_{\alpha}}\left(x_{\alpha}\right)}\left|\nabla_{g} u_{\alpha}\right|^{2} \leq C \mu_{\alpha}^{n-2} .
$$


Proof. Let $\eta$ be a cut-off function satisfying $S u p p(\eta) \subset M \backslash B_{\delta / 2}\left(x_{\alpha}\right)$ and $\eta \equiv 1$ in $M \backslash B_{\delta}\left(x_{\alpha}\right)$. Multiplying the Euler-Lagrange equation (2.2) by $\eta^{2} u_{\alpha}$ and integrating by parts, we have

$$
\int_{M} \nabla_{g} u_{\alpha} \nabla_{g}\left(\eta^{2} u_{\alpha}\right) \mathrm{d} v_{g} \leq-\frac{n-2}{2} \int_{\partial M} h \eta^{2} u_{\alpha}^{2} \mathrm{~d} s_{g}+\ell_{\alpha} \int_{\partial M} \eta^{2} u_{\alpha}^{q} \mathrm{~d} s_{g} .
$$

It follows that

$$
\int_{M} \eta^{2}\left|\nabla_{g} u_{\alpha}\right|^{2} \mathrm{~d} v_{g} \leq C \int_{\partial M}\left(\eta^{2} u_{\alpha}^{2}+\eta^{2} u_{\alpha}^{q}\right) \mathrm{d} s_{g}+C \int_{M}\left|\nabla_{g} \eta\right|^{2} u_{\alpha}^{2} \mathrm{~d} v_{g} .
$$

Therefore, this corollary follows immediately from Proposition 2.3 ,

\section{Energy estimates And Proof of Theorem 1.2}

For some small $\delta_{0}$ to be determined in Lemma 3.4. let $\psi_{\alpha} \in C^{\infty}(\bar{M})$ satisfy $\psi_{\alpha}\left(x_{\alpha}\right)=1, \frac{1}{2} \leq \psi_{\alpha} \leq 2,\left\|\psi_{\alpha}\right\|_{C^{2}(\bar{M})} \leq C$, and

$$
\begin{cases}\Delta_{g} \psi_{\alpha}=0, & \text { in } B_{2 \delta_{0}}^{+} \\ \frac{\partial_{g} \psi_{\alpha}}{\partial \nu}+\frac{n-2}{2} h_{g} \psi_{\alpha}=0, & \text { on } \partial^{\prime} B_{2 \delta_{0}}^{+}\end{cases}
$$

As in the previous section, here we used the Fermi coordinate with respect to metric $g$ centered at $x_{\alpha}$. Set $\hat{g}=\psi_{\alpha}^{4 /(n-2)} g$. It is easy to see that $h_{\hat{g}}=0$ on $\partial^{\prime} B_{\delta_{0}}^{+}$. Hence, $u_{\alpha} / \psi_{\alpha}$ satisfies

$$
\begin{cases}\Delta_{\hat{g}} \frac{u_{\alpha}}{\psi_{\alpha}}=0, & \text { in } B_{2 \delta_{0}}^{+}, \\ \frac{\partial_{\hat{g}}}{\partial \nu} \frac{u_{\alpha}}{\psi_{\alpha}}+\alpha\left\|u_{\alpha}\right\|_{L^{r}(\partial M)}^{2-r} \psi_{\alpha}^{1-q} u_{\alpha}^{r-1}=\ell_{\alpha}\left(\frac{u_{\alpha}}{\psi_{\alpha}}\right)^{q-1}, & \text { on } \partial^{\prime} B_{2 \delta_{0}}^{+} .\end{cases}
$$

It follows that the maximum of $u_{\alpha} / \psi_{\alpha}$ on $\bar{M}$ is achieved at some point on $\partial M$ which is denoted as $\hat{x}_{\alpha}$. In view of the fact $u_{\alpha}\left(x_{\alpha}\right) \rightarrow \infty$ and Proposition 2.3, we have $\left|\hat{x}_{\alpha}-x_{\alpha}\right| \rightarrow 0$ and thus $\frac{u_{\alpha}\left(\hat{x}_{\alpha}\right)}{\psi_{\alpha}\left(\hat{x}_{\alpha}\right) u_{\alpha}\left(x_{\alpha}\right)} \rightarrow 1$ as $\alpha \rightarrow \infty$. From now on, we use the Fermi coordinate with respect to the metric $\hat{g}$ centered at $x_{\alpha}$. Since $\psi_{\alpha}\left(x_{\alpha}\right)=1$, by a simple blow-up argument and the same proof of Proposition 2.2 we can establish

$$
\lim _{\alpha \rightarrow \infty}\left(\int_{B_{\delta}^{+}}\left|\nabla_{\hat{g}}\left(\frac{u_{\alpha}}{\psi_{\alpha}}-U_{\mu_{\alpha}}\right)\right|^{2} \mathrm{~d} v_{\hat{g}}+\int_{\partial^{\prime} B_{\delta}^{+}}\left|\frac{u_{\alpha}}{\psi_{\alpha}}-U_{\mu_{\alpha}}\right|^{q} \mathrm{~d} s_{\hat{g}}\right)=0 .
$$

As in Corollary 2.1, we can select $\delta_{\alpha} \in\left[\delta_{0} / 2, \delta_{0}\right]$ such that

$$
\int_{\partial^{\prime \prime} B_{\delta_{\alpha}}}\left|\nabla_{\hat{g}}\left(\frac{u_{\alpha}}{\psi_{\alpha}}\right)\right|^{2} \mathrm{~d} v_{\hat{g}} \leq C \mu_{\alpha}^{n-2},
$$

where $C>0$ is independent of $\alpha$. Let us focus on the upper half ball $B_{\delta_{\alpha}}^{+}(0)$, which is equipped with the Riemannian metric $\hat{g}$.

Let $h_{\xi, \lambda}(z), z \in \bar{B}_{\delta_{\alpha}}^{+}(0)$, be the classical solution of

$$
\begin{cases}\Delta_{\hat{g}} h_{\xi, \lambda}=0 & \text { in } B_{\delta_{\alpha}}^{+}, \\ h_{\xi, \lambda}=U_{\xi, \lambda} & \text { on } \partial^{\prime \prime} B_{\delta_{\alpha}}^{+}, \\ \frac{\partial_{\hat{g}} h_{\xi, \lambda}}{\partial \nu}=0 & \text { on } \partial^{\prime} B_{\delta_{\alpha}}^{+},\end{cases}
$$


with parameters $\xi \in \partial^{\prime} B_{\delta_{\alpha} \mu_{\alpha} / 2}^{+}$and $\lambda>0$, and let $\chi_{\alpha}(z)$ be the solution of

$$
\begin{cases}\Delta_{\hat{g}} \chi_{\alpha}=0 & \text { in } B_{\delta_{\alpha}}^{+}, \\ \chi_{\alpha}=\frac{u_{\alpha}}{\psi_{\alpha}} & \text { on } \partial^{\prime \prime} B_{\delta_{\alpha}}, \\ \frac{\partial_{\hat{g}} \chi_{\alpha}}{\partial \nu}=0 & \text { on } \partial^{\prime} B_{\delta_{\alpha}}^{+} .\end{cases}
$$

Then $u_{\alpha} / \psi_{\alpha}-\chi_{\alpha} \in H_{0, L}\left(B_{\delta_{\alpha}}^{+}\right)$and $U_{\xi, \lambda}-h_{\xi, \lambda} \in H_{0, L}\left(B_{\delta_{\alpha}}^{+}\right)$are the projections of $u_{\alpha} / \psi_{\alpha}$ and $U_{\xi, \lambda}$ on $H_{0, L}\left(B_{\delta_{\alpha}}\right)$, respectively. Here

$$
H_{0, L}\left(B_{\delta_{\alpha}}^{+}\right):=\left\{u \in H^{1}\left(B_{\delta_{\alpha}}^{+}\right): u=0 \text { on } \partial^{\prime \prime} B_{\delta_{\alpha}}^{+} \text {in trace sense }\right\}
$$

is a Hilbert space with the inner product $\langle u, v\rangle_{\hat{g}}:=\int_{B_{\delta_{\alpha}}^{+}} \nabla_{\hat{g}} u \nabla_{\hat{g}} v \mathrm{~d} v_{\hat{g}}$. Denote $\|u\|=\sqrt{\langle u, u\rangle_{\hat{g}}}$, which is a norm for $u \in H_{0, L}\left(B_{\delta_{\alpha}}^{+}\right)$.

Set

$$
\sigma_{\xi, \lambda}=U_{\xi, \lambda}-h_{\xi, \lambda},
$$

which satisfies $\sigma_{\xi, \lambda} \leq U_{\xi, \lambda}$ and

$$
\begin{cases}\Delta_{\hat{g}} \sigma_{\xi, \lambda}=\Delta_{\hat{g}} U_{\xi, \lambda}, & \text { in } B_{\delta_{\alpha}}^{+}, \\ \sigma_{\xi, \lambda}=0, & \text { on } \partial^{\prime \prime} B_{\delta_{\alpha}}^{+}, \\ \frac{\partial_{\hat{g}} \sigma_{\xi, \lambda}}{\partial \nu}=\frac{\partial_{\hat{g}} U_{\xi, \lambda}}{\partial \nu} & \text { on } \partial^{\prime} B_{\delta_{\alpha}}^{+} .\end{cases}
$$

Let $\left(t_{\alpha}, \xi_{\alpha}, \lambda_{\alpha}\right) \in\left[\frac{1}{2}, \frac{3}{2}\right] \times \overline{\partial^{\prime} B_{\delta_{\alpha} \mu_{\alpha} / 2}^{+}} \times\left[\frac{\mu_{\alpha}}{2}, \frac{3 \mu_{\alpha}}{2}\right]$ be such that

$$
\begin{aligned}
& \left\|\frac{u_{\alpha}}{\psi_{\alpha}}-\chi_{\alpha}-t_{\alpha} \sigma_{\xi_{\alpha}, \lambda_{\alpha}}\right\| \\
& =\min \left\{\left\|\frac{u_{\alpha}}{\psi_{\alpha}}-\chi_{\alpha}-t \sigma_{\xi, \lambda}\right\|:(t, \xi, \lambda) \in\left[\frac{1}{2}, \frac{3}{2}\right] \times \overline{\partial^{\prime} B_{\delta_{\alpha} \mu_{\alpha} / 2}^{+}} \times\left[\frac{\mu_{\alpha}}{2}, \frac{3 \mu_{\alpha}}{2}\right]\right\},
\end{aligned}
$$

and

Define

$$
w_{\alpha}=\frac{u_{\alpha}}{\psi_{\alpha}}-\chi_{\alpha}-t_{\alpha} \sigma_{\xi_{\alpha}, \lambda_{\alpha}}
$$

$W_{\alpha}=\left\{w \in H_{0, L}\left(B_{\delta_{\alpha}}^{+}\right):\left\langle\sigma_{\xi_{\alpha}, \lambda_{\alpha}}, w\right\rangle_{\hat{g}}=0\right.$ and $\langle u, w\rangle_{\hat{g}}=0$ for all $\left.u \in E_{\alpha}\right\}$,

where $E_{\alpha} \subset H_{0, L}\left(B_{\delta_{\alpha}}^{+}\right)$is the tangent space at $\sigma_{\xi_{\alpha}, \lambda_{\alpha}}$ of the $n$-dimensional surface $\left\{\sigma_{\xi, \lambda}: \xi \in \partial^{\prime} B_{\mu_{\alpha} \delta_{\alpha}}, \lambda>0\right\} \subset H_{0, L}\left(B_{\delta_{\alpha}}^{+}\right)$. More explicitly,

$$
E_{\alpha}=\operatorname{span}\left\{\left.\frac{\partial \sigma_{\xi, \lambda_{\alpha}}}{\partial \xi}\right|_{\xi=\xi_{\alpha}},\left.\frac{\partial \sigma_{\xi_{\alpha}, \lambda}}{\partial \lambda}\right|_{\lambda=\lambda_{\alpha}}\right\} .
$$

For brevity, we denote $h_{\alpha}=h_{\xi_{\alpha}, \lambda_{\alpha}}$ and $\sigma_{\alpha}=\sigma_{\xi_{\alpha}, \lambda_{\alpha}}$.

Lemma 3.1. We have

1) $\left\|\nabla_{\hat{g}} h_{\alpha}\right\|_{L^{2}\left(B_{\delta_{\alpha}}\right)}+\left\|h_{\alpha}\right\|_{L^{\infty}\left(B_{\delta_{\alpha}}\right)} \leq C \mu_{\alpha}^{(n-2) / 2}$,

2) $\left\|\nabla_{\hat{g}} \chi_{\alpha}\right\|_{L^{2}\left(B_{\delta_{\alpha}}\right)}+\left\|\chi_{\alpha}\right\|_{L^{\infty}\left(B_{\delta_{\alpha}}\right)} \leq C \mu_{\alpha}^{(n-2) / 2}$,

for some positive constant $C$ independent of $\alpha$, and

3) $\left\|w_{\alpha}\right\| \rightarrow 0$,

4) $t_{\alpha} \rightarrow 1$,

5) $\mu_{\alpha}^{-1}\left|\xi_{\alpha}\right| \rightarrow 0$

6) $\mu_{\alpha}^{-1} \lambda_{\alpha} \rightarrow 1$,

as $\alpha \rightarrow \infty$. Furthermore, $w_{\alpha} \in W_{\alpha}$. 
Proof. Let $\eta \in H^{1}\left(B_{\delta_{\alpha}}\right)$ be an extension of $\left.U_{\xi_{\alpha}, \lambda_{\alpha}}\right|_{\partial^{\prime \prime} B_{\delta_{\alpha}}^{+}}$such that

$$
\|\eta\|_{H^{1}\left(B_{\delta_{\alpha}}\right)}^{2} \leq C\left(\int_{\partial^{\prime \prime} B_{\delta_{\alpha}}^{+}}\left|\nabla U_{\xi_{\alpha}, \lambda_{\alpha}}\right|^{2}+U_{\xi_{\alpha}, \lambda_{\alpha}}^{2}\right)
$$

where $C>0$ is independent of $\alpha$. Multiplying the equation of $h_{\alpha}$ by $h_{\alpha}-\eta \in$ $H_{0, L}\left(B_{\delta_{\alpha}}\right)$ and integrating by parts we have

$$
0=\int_{B_{\delta_{\alpha}}} \nabla_{\hat{g}} h_{\alpha} \nabla_{\hat{g}}\left(h_{\alpha}-\eta\right) \mathrm{d} v_{\hat{g}} \geq \frac{1}{2}\left(\left\|\nabla_{\hat{g}} h_{\alpha}\right\|_{L^{2}}^{2}-\left\|\nabla_{\hat{g}} \eta\right\|_{L^{2}}^{2}\right) .
$$

Thus we obtained the $L^{2}$ estimate for $\nabla_{\hat{g}} h_{\alpha}$. The $L^{\infty}$ estimates for $h_{\alpha}$ follow easily from the maximum principle. Hence, we verified 1). Similarly, we can verify 2) by taking into account Proposition 2.3 and (3.3).

By the definition of $t_{\alpha}$ and $\sigma_{\alpha}$,

$$
\begin{aligned}
\left\|t_{\alpha} \sigma_{\alpha}-\sigma_{0, \mu_{\alpha}}\right\| & \leq\left\|\frac{u_{\alpha}}{\psi_{\alpha}}-\chi_{\alpha}-t_{\alpha} \sigma_{\alpha}\right\|+\left\|\frac{u_{\alpha}}{\psi_{\alpha}}-\chi_{\alpha}-\sigma_{0, \mu_{\alpha}}\right\| \\
& \leq 2\left\|\frac{u_{\alpha}}{\psi_{\alpha}}-\chi_{\alpha}-\sigma_{0, \mu_{\alpha}}\right\| \\
& \leq 2\left\|\frac{u_{\alpha}}{\psi_{\alpha}}-U_{\mu_{\alpha}}\right\|+C \mu_{\alpha}^{(n-2) / 2} \rightarrow 0
\end{aligned}
$$

as $\alpha \rightarrow \infty$, where we used 1), 2), and (3.2). It follows that $\left\|w_{\alpha}\right\| \rightarrow 0$, i.e., 3) holds, and

$$
\left\|t_{\alpha} U_{\xi_{\alpha}, \lambda_{\alpha}}-U_{\mu_{\alpha}}\right\| \leq\left\|t_{\alpha} \sigma_{\alpha}-\sigma_{0, \mu_{\alpha}}\right\|+\left\|t_{\alpha} h_{\xi_{\alpha}, \lambda_{\alpha}}\right\|+\left\|h_{0, \mu_{\alpha}}\right\| \rightarrow 0
$$

as $\alpha \rightarrow \infty$. A simple calculation yields 4), 5), and 6). Once we have 4), 5), and 6), the minimum of the norm is attained in the interior of $\left[\frac{1}{2}, \frac{3}{2}\right] \times \overline{\partial^{\prime} B_{\delta_{\alpha} \mu_{\alpha} / 2}^{+}} \times\left[\frac{\mu_{\alpha}}{2}, \frac{3 \mu_{\alpha}}{2}\right]$. Hence, a variational argument gives $w_{\alpha} \in W_{\alpha}$.

Proposition 3.1. Assume as above that we have

$$
\begin{aligned}
\left\|w_{\alpha}\right\|+\left|t_{\alpha}^{q-2} \ell_{\alpha}-S^{-1}\right| \leq C\{ & \mu_{\alpha}\left\|U_{1}^{\frac{n-1}{n-2}}\right\|_{L^{2^{*^{\prime}}}\left(B_{\mu_{\alpha}^{-1}}^{+}\right)} \\
& \left.+\epsilon_{\alpha}\left\|U_{1}^{r-1}\right\|_{L^{r}\left(\partial^{\prime} B_{\mu_{\alpha}^{-1}}^{+}\right)}+\mu_{\alpha}^{n-2}\left\|U_{1}^{q-2}\right\|_{L^{r}\left(\partial^{\prime} B_{\mu_{\alpha}^{-1}}^{+}\right)}\right\},
\end{aligned}
$$

where $\epsilon_{\alpha}$ is given in (2.8), $2^{*}=\frac{2 n}{n-2}$ and $2^{*^{\prime}}=\frac{2 n}{n+2}$.

We postpone the proof of Proposition 3.1 to the end of this section, and use it to prove Theorem 1.2 first.

Let

$$
Y\left(g, u_{\alpha}\right)=\frac{\int_{M}\left|\nabla_{g} u_{\alpha}\right|^{2} \mathrm{~d} v_{g}+\frac{n-2}{2} \int_{\partial M} h_{g} u_{\alpha}^{2} \mathrm{~d} s_{g}}{\left(\int_{\partial M} u_{\alpha}^{q} \mathrm{~d} s_{g}\right)^{2 / q}} .
$$

It follows from Proposition 2.3 that

$$
Y\left(g, u_{\alpha}\right)=\frac{\int_{B_{\delta_{\alpha}}^{+}}\left|\nabla_{g} u_{\alpha}\right|^{2} \mathrm{~d} v_{g}+\frac{n-2}{2} \int_{\partial^{\prime} B_{\delta_{\alpha}}^{+}} h_{g} u_{\alpha}^{2} \mathrm{~d} s_{g}}{\left(\int_{\partial^{\prime} B_{\delta_{\alpha}}^{+}} u_{\alpha}^{q} \mathrm{~d} s_{g}\right)^{2 / q}}+O\left(\mu_{\alpha}^{n-2}\right) .
$$

Since

$$
\left\{\begin{array}{l}
\Delta_{g} u_{\alpha}-c(n) R_{g} u_{\alpha}=\varphi_{\alpha}^{\frac{n+2}{n-2}}\left(\Delta_{\hat{g}} \frac{u_{\alpha}}{\varphi_{\alpha}}-c(n) R_{\hat{g}} \frac{u_{\alpha}}{\varphi_{\alpha}}\right) \quad \text { on } M \\
\frac{\partial_{g} u_{\alpha}}{\partial \nu}+\frac{n-2}{2} h_{g} u_{\alpha}=\varphi_{\alpha}^{\frac{n}{n-2}}\left(\frac{\partial_{\hat{g}}}{\partial \nu} \frac{u_{\alpha}}{\varphi_{\alpha}}+\frac{n-2}{2} h_{\hat{g}} \frac{u_{\alpha}}{\varphi_{\alpha}}\right) \quad \text { on } \partial M
\end{array}\right.
$$


and $h_{\hat{g}}=0$ on $\partial^{\prime} B_{\delta_{\alpha}}^{+}$, we have

$$
Y\left(g, u_{\alpha}\right)=\frac{\int_{B_{\delta_{\alpha}}^{+}}\left|\nabla_{\hat{g}}\left(\frac{u_{\alpha}}{\varphi_{\alpha}}\right)\right|^{2} \mathrm{~d} v_{\hat{g}}}{\left(\int_{\partial^{\prime} B_{\delta_{\alpha}}^{+}}\left(\frac{u_{\alpha}}{\varphi_{\alpha}}\right)^{q} \mathrm{~d} s_{\hat{g}}\right)^{2 / q}}+O\left(\mu_{\alpha}^{n-2}\right) .
$$

In view of $u_{\alpha} / \varphi_{\alpha}=t_{\alpha} U_{\xi_{\alpha}, \lambda_{\alpha}}-t_{\alpha} h_{\alpha}+\chi_{\alpha}+w_{\alpha}$,

$$
\int_{B_{\delta_{\alpha}}^{+}} \nabla_{\hat{g}} \chi_{\alpha} \nabla_{\hat{g}} w_{\alpha} \mathrm{d} v_{\hat{g}}=\int_{B_{\delta_{\alpha}}^{+}} \nabla_{\hat{g}} h_{\alpha} \nabla_{\hat{g}} w_{\alpha} \mathrm{d} v_{\hat{g}}=0
$$

and the estimates in Lemma 3.1, we have

$$
Y\left(g, u_{\alpha}\right)=F\left(w_{\alpha}\right)+O\left(\mu_{\alpha}^{n-2}\right),
$$

where

By a direct computation,

$$
F(w):=\frac{\int_{B_{\delta_{\alpha}}^{+}}\left|\nabla_{\hat{g}}\left(t_{\alpha} U_{\xi_{\alpha}, \lambda_{\alpha}}+w\right)\right|^{2} \mathrm{~d} v_{\hat{g}}}{\left(\int_{\partial^{\prime} B_{\delta_{\alpha}}^{+}}\left|t_{\alpha} U_{\xi_{\alpha}, \lambda_{\alpha}}+w\right|^{q} \mathrm{~d} s_{\hat{g}}\right)^{2 / q}} .
$$

$$
\begin{aligned}
F^{\prime}(0) w_{\alpha}= & \frac{2}{\left(\int_{\partial^{\prime} B_{\delta_{\alpha}}^{+}}\left|t_{\alpha} U_{\xi_{\alpha}, \lambda_{\alpha}}\right| q \mathrm{~d} s_{\hat{g}}\right)^{2 / q}}\left\{\int_{B_{\delta_{\alpha}}^{+}} t_{\alpha} \nabla_{\hat{g}} U_{\xi_{\alpha}, \lambda_{\alpha}} \nabla_{\hat{g}} w_{\alpha} \mathrm{d} v_{\hat{g}}\right. \\
& \left.-\frac{\int_{B_{\delta_{\alpha}}^{+}}\left|t_{\alpha} \nabla_{\hat{g}} U_{\xi_{\alpha}, \lambda_{\alpha}}\right|^{2} \mathrm{~d} v_{\hat{g}}}{\int_{\partial^{\prime} B_{\delta_{\alpha}}^{+}}\left|t_{\alpha} U_{\xi_{\alpha}, \lambda_{\alpha}}\right|^{q} \mathrm{~d} s_{\hat{g}}} \int_{\partial^{\prime} B_{\delta_{\alpha}}^{+}}\left|t_{\alpha} U_{\xi_{\alpha}, \lambda_{\alpha}}\right|^{q-1} w_{\alpha} \mathrm{d} s_{\hat{g}}\right\} .
\end{aligned}
$$

Recall that $\sigma_{\alpha}=U_{\xi_{\alpha}, \lambda_{\alpha}}-h_{\alpha}$ and $\int_{B_{\delta_{\alpha}}^{+}} \nabla_{\hat{g}} \sigma_{\alpha} \nabla_{\hat{g}} w_{\alpha}=\int_{B_{\delta_{\alpha}}^{+}} \nabla_{\hat{g}} h_{\alpha} \nabla_{\hat{g}} w_{\alpha}=0$. It follows that $\int_{B_{\delta_{\alpha}}^{+}} \nabla_{\hat{g}} U_{\xi_{\alpha}, \lambda_{\alpha}} \nabla_{\hat{g}} w_{\alpha}=0$. Hence (recall that we are in the Fermi coordinates of $\hat{g}$ ),

$$
\begin{aligned}
\left|F^{\prime}(0) w_{\alpha}\right| & \leq\left. C\left|\int_{\partial^{\prime} B_{\delta_{\alpha}}^{+}}\right| U_{\xi_{\alpha}, \lambda_{\alpha}}\right|^{q-1} w_{\alpha} \mathrm{d} s_{\hat{g}}|=C S| \int_{\partial^{\prime} B_{\delta_{\alpha}}^{+}} \frac{\partial_{\hat{g}}}{\partial \nu} U_{\xi_{\alpha}, \lambda_{\alpha}} w_{\alpha} \mathrm{d} s_{\hat{g}} \mid \\
& =C S\left|\int_{B_{\delta_{\alpha}}^{+}} \Delta_{\hat{g}} U_{\xi_{\alpha}, \lambda_{\alpha}} w_{\alpha} \mathrm{d} v_{\hat{g}}\right| \\
& \leq C\left\|\Delta_{\hat{g}} U_{\xi_{\alpha}, \lambda_{\alpha}}\right\|_{L^{2^{*^{\prime}}}\left(B_{\delta_{\alpha}}^{+}\right)}\left\|w_{\alpha}\right\|,
\end{aligned}
$$

where $2^{*}=\frac{2 n}{n-2}$ and $2^{*^{\prime}}=\frac{2 n}{n+2}$. By (3.9) we have

$$
\left|F^{\prime}(0) w_{\alpha}\right| \leq C \mu_{\alpha}\left\|U_{1}^{\frac{n-1}{n-2}}\right\|_{L^{2^{*^{\prime}}\left(B_{\mu_{\alpha}}^{+}\right)}}\left\|w_{\alpha}\right\| .
$$

Similarly,

$$
\begin{aligned}
\left\langle F^{\prime \prime}(0) w_{\alpha}, w_{\alpha}\right\rangle= & \frac{2}{\left(\int_{\partial^{\prime} B_{\delta_{\alpha}}^{+}}\left|t_{\alpha} U_{\xi_{\alpha}, \lambda_{\alpha}}\right|^{q} \mathrm{~d} s_{\hat{g}}\right)^{2 / q}}\left\{\int_{B_{\delta_{\alpha}}^{+}}\left|\nabla_{\hat{g}} w_{\alpha}\right|^{2} \mathrm{~d} v_{\hat{g}}\right. \\
& \left.-(q-1) \frac{\int_{B_{\delta_{\alpha}}^{+}}\left|t_{\alpha} \nabla_{\hat{g}} U_{\xi_{\alpha}, \lambda_{\alpha}}\right|^{2} \mathrm{~d} v_{\hat{g}}}{\int_{\partial^{\prime} B_{\delta_{\alpha}}^{+}}\left|t_{\alpha} U_{\xi_{\alpha}, \lambda_{\alpha}}\right|^{q} \mathrm{~d} s_{\hat{g}}} \int_{\partial^{\prime} B_{\delta_{\alpha}}^{+}}\left|t_{\alpha} U_{\xi_{\alpha}, \lambda_{\alpha}}\right|^{q-2} w_{\alpha}^{2} \mathrm{~d} s_{\hat{g}}\right\} \\
& +O\left(\left(\int_{\partial^{\prime} B_{\delta_{\alpha}}^{+}}\left|U_{\xi_{\alpha}, \lambda_{\alpha}}\right|^{q-1} w_{\alpha} \mathrm{d} s_{\hat{g}}\right)^{2}\right) .
\end{aligned}
$$


By Lemma 3.6, we have

$$
\begin{aligned}
& \int_{B_{\delta_{\alpha}}^{+}}\left|\nabla_{\hat{g}} w_{\alpha}\right|^{2} \mathrm{~d} v_{\hat{g}}-\frac{(q-1) \int_{B_{\delta_{\alpha}}^{+}}\left|t_{\alpha} \nabla_{\hat{g}} U_{\xi_{\alpha}, \lambda_{\alpha}}\right|^{2} \mathrm{~d} v_{\hat{g}}}{\int_{\partial^{\prime} B_{\delta_{\alpha}}^{+}}\left|t_{\alpha} U_{\xi_{\alpha}, \lambda_{\alpha}}\right|^{q} \mathrm{~d} s_{\hat{g}}} \int_{\partial^{\prime} B_{\delta_{\alpha}}^{+}}\left|t_{\alpha} U_{\xi_{\alpha}, \lambda_{\alpha}}\right|^{q-2} w_{\alpha}^{2} \mathrm{~d} s_{\hat{g}} \\
& \quad \geq \frac{c_{1}}{2}\left\|w_{\alpha}\right\|^{2},
\end{aligned}
$$

for large $\alpha$. It follows that

$$
\left\langle F^{\prime \prime}(0) w_{\alpha}, w_{\alpha}\right\rangle \geq \frac{c_{1}}{2}\left\|w_{\alpha}\right\|^{2}+O\left(\mu_{\alpha}^{2}\right)\left\|U_{1}^{\frac{n-1}{n-2}}\right\|_{L^{2^{*^{\prime}}\left(B_{\mu_{\alpha}}^{+}\right)}}^{2}\left\|w_{\alpha}\right\|^{2} .
$$

Noticing that $\mu_{\alpha}\left\|U_{1}^{\frac{n-1}{n-2}}\right\|_{L^{2^{*^{\prime}}\left(B_{\mu_{\alpha}}^{+}\right)}}^{2} \rightarrow 0$ as $\alpha \rightarrow \infty$, we have

$$
\begin{aligned}
F\left(w_{\alpha}\right) & =F(0)+F^{\prime}(0) w_{\alpha}+\frac{1}{2}\left\langle F^{\prime \prime}(0) w_{\alpha}, w_{\alpha}\right\rangle+o\left(\left\|w_{\alpha}\right\|^{2}\right) \\
& \geq F(0)+O\left(\mu_{\alpha}\left\|U_{1}^{\frac{n-1}{n-2}}\right\|_{L^{2^{*^{\prime}}\left(B_{\mu_{\alpha}}^{+}\right)}}\left\|w_{\alpha}\right\|\right) .
\end{aligned}
$$

By (3.5), we conclude that

(3.6) $Y\left(g, u_{\alpha}\right) \geq \frac{\int_{B_{\delta_{\alpha}}^{+}}\left|\nabla_{\hat{g}} U_{\xi_{\alpha}, \lambda_{\alpha}}\right|^{2} \mathrm{~d} v_{\hat{g}}}{\left(\int_{\partial^{\prime} B_{\delta_{\alpha}}^{+}} U_{\xi_{\alpha}, \lambda_{\alpha}}^{q} \mathrm{~d} s_{\hat{g}}\right)^{2 / q}}+O\left(\mu_{\alpha}\left\|U_{1}^{\frac{n-1}{n-2}}\right\|_{L^{2^{*^{\prime}}}\left(B_{\mu_{\alpha}}^{+}\right)}\left\|w_{\alpha}\right\|+\mu_{\alpha}^{n-2}\right)$.

Lemma 3.2. We have

$$
\frac{\int_{B_{\delta_{\alpha}}^{+}}\left|\nabla_{\hat{g}} U_{\xi_{\alpha}, \lambda_{\alpha}}\right|^{2} \mathrm{~d} v_{\hat{g}}}{\left(\int_{\partial^{\prime} B_{\delta_{\alpha}}^{+}} U_{\xi_{\alpha}, \lambda_{\alpha}}^{q} \mathrm{~d} s_{\hat{g}}\right)^{2 / q}}= \begin{cases}\frac{1}{S}+O\left(\mu_{\alpha}^{2}\right), & n \geq 5, \\ \frac{1}{S}+O\left(\mu_{\alpha}^{2} \log \mu_{\alpha}^{-1}\right), & n=4 .\end{cases}
$$

Proof. Let $\pi=\hat{h}_{i j} \mathrm{~d} z^{i} \mathrm{~d} z^{j}, 1 \leq i, j \leq n-1$, be the second fundamental form of $\partial^{\prime} B_{\delta_{\alpha}}^{+}$with respect to the metric $\hat{g}_{i j}$. Since $\sqrt{\operatorname{det} \hat{g}_{i j}}=1+O\left(|z|^{2}\right)$ on $\partial^{\prime} B_{\delta_{\alpha}}^{+}$, we have

$$
\begin{aligned}
\left(\int_{\partial^{\prime} B_{\delta_{\alpha}}^{+}} U_{\xi_{\alpha}, \lambda_{\alpha}}^{q} \mathrm{~d} s_{\hat{g}}\right)^{2 / q}= & \left(\int_{\partial^{\prime} B_{\delta_{\alpha}}^{+}} U_{\xi_{\alpha}, \lambda_{\alpha}}^{q}\left(1+O\left(\left|z^{\prime}\right|^{2}\right)\right) \mathrm{d} z\right)^{2 / q} \\
= & \left(\int_{\partial^{\prime} B_{\delta_{\alpha}}^{+}} U_{\xi_{\alpha}, \lambda_{\alpha}}^{q} \mathrm{~d} z\right)^{2 / q}+O\left(\int_{\partial^{\prime} B_{\delta_{\alpha}}^{+}} U_{\xi_{\alpha}, \lambda_{\alpha}}^{q}\left|z^{\prime}\right|^{2} \mathrm{~d} z\right) \\
= & \left(\int_{\partial \mathbb{R}_{+}^{n}} U_{\xi_{\alpha}, \lambda_{\alpha}}^{q} \mathrm{~d} z\right)^{2 / q}+O\left(\lambda_{\alpha}^{n-2}\right) \\
& +O\left(\lambda_{\alpha}^{2} \int_{\partial^{\prime} B_{\lambda_{\alpha}^{-1}}^{+}}\left(1+\left|z^{\prime}\right|^{2}\right)^{1-n}\left|z^{\prime}\right|^{2} \mathrm{~d} z\right) \\
= & 1+O\left(\lambda_{\alpha}^{n-2}\right)+O\left(\lambda_{\alpha}^{2} \int_{\partial^{\prime} B_{\lambda_{\alpha}^{-1}}^{+}}\left(1+\left|z^{\prime}\right|^{2}\right)^{1-n}\left|z^{\prime}\right|^{2} \mathrm{~d} z\right),
\end{aligned}
$$

where we used $\lambda_{\alpha}^{-1}\left|\xi_{\alpha}\right| \rightarrow 0$ as $\alpha \rightarrow \infty$ in the last equality. 
In addition, by Lemma 2.2, we have

$$
\begin{aligned}
\int_{B_{\delta_{\alpha}}^{+}}\left|\nabla_{\hat{g}} U_{\xi_{\alpha}, \lambda_{\alpha}}\right|^{2} \mathrm{~d} v_{\hat{g}}= & \int_{B_{\delta_{\alpha}}^{+}}\left|\nabla U_{\xi_{\alpha}, \lambda_{\alpha}}\right|^{2} \mathrm{~d} z+2 \hat{h}^{i j}(0) \int_{B_{\delta_{\alpha}}^{+}} \partial_{i} U_{\xi_{\alpha}, \lambda_{\alpha}} \partial_{j} U_{\xi_{\alpha}, \lambda_{\alpha}} z_{n} \mathrm{~d} z \\
& +O\left(\int_{B_{\delta_{\alpha}}^{+}}\left|\nabla U_{\xi_{\alpha}, \lambda_{\alpha}}\right|^{2}|z|^{2} \mathrm{~d} z\right) .
\end{aligned}
$$

It is easy to see that

$$
\int_{B_{\delta_{\alpha}}^{+}}\left|\nabla U_{\xi_{\alpha}, \lambda_{\alpha}}\right|^{2} \mathrm{~d} z=\int_{\mathbb{R}_{+}^{n}}\left|\nabla U_{\xi_{\alpha}, \lambda_{\alpha}}\right|^{2} \mathrm{~d} z+O\left(\lambda_{\alpha}^{n-2}\right)=\frac{1}{S}+O\left(\lambda_{\alpha}^{n-2}\right)
$$

and

$$
\begin{aligned}
& \int_{B_{\delta_{\alpha}}^{+}}\left|\nabla U_{\xi_{\alpha}, \lambda_{\alpha}}\right|^{2}|z|^{2} \mathrm{~d} z \\
& \quad=C(n) \lambda_{\alpha}^{2} \int_{B_{\lambda_{\alpha}^{-1}}^{+}}\left(\left|z^{\prime}\right|^{2}+\left(z_{n}+1\right)^{2}\right)^{1-n}\left|z+\lambda_{\alpha}^{-1} \xi_{\alpha}\right|^{2} \mathrm{~d} z+O\left(\lambda_{\alpha}^{n-2}\right) .
\end{aligned}
$$

By symmetry, we have

$$
\begin{aligned}
& \sum_{i, j=1}^{n-1} \hat{h}^{i j}(0) \int_{B_{\delta_{\alpha}}^{+}} \partial_{i} U_{\xi_{\alpha}, \lambda_{\alpha}} \partial_{j} U_{\xi_{\alpha}, \lambda_{\alpha}} z_{n} \mathrm{~d} z \\
& =\sum_{i, j=1}^{n-1} \hat{h}^{i j}(0) \int_{B_{\delta_{\alpha} / 2}^{+}\left(\xi_{\alpha}\right)} \partial_{i} U_{\xi_{\alpha}, \lambda_{\alpha}} \partial_{j} U_{\xi_{\alpha}, \lambda_{\alpha}} z_{n} \mathrm{~d} z+O\left(\mu_{\alpha}^{n-2}\right) \\
& =\sum_{i=1}^{n-1} \hat{h}^{i i}(0) \int_{B_{\delta_{\alpha} / 2}^{+}\left(\xi_{\alpha}\right)}\left|\partial_{1} U_{\xi_{\alpha}, \lambda_{\alpha}}\right|^{2} z_{n} \mathrm{~d} z+O\left(\mu_{\alpha}^{n-2}\right)=O\left(\mu_{\alpha}^{n-2}\right),
\end{aligned}
$$

where we used $\sum_{i=1}^{n-1} \hat{h}^{i i}(0)=0$ since the mean curvature of $\hat{g}$ is vanishing at $x_{\alpha}$. The lemma follows immediately from Lemma 3.1

Proof of Theorem 1.2. Notice that

$$
\frac{1}{S}>I_{\alpha}\left(u_{\alpha}\right)=Y\left(g, u_{\alpha}\right)+\alpha\left\|u_{\alpha}\right\|_{L^{r}(\partial M)}^{2} .
$$

By (3.6) and Lemma 3.2 we have

$$
\alpha\left\|u_{\alpha}\right\|_{L^{r}(\partial M)}^{2}=O\left(\mu_{\alpha}^{2}\right)+O\left(\mu_{\alpha}\left\|U_{1}^{\frac{n-1}{n-2}}\right\|_{L^{2^{*^{\prime}}}\left(B_{\mu_{\alpha}^{-1}}^{+}\right)}\left\|w_{\alpha}\right\|+\mu_{\alpha}^{n-2}\right) .
$$

By Proposition 3.1, we find

$$
\begin{aligned}
\alpha\left\|u_{\alpha}\right\|_{L^{r}(\partial M)}^{2} \leq C\left\{\mu_{\alpha}\left\|U_{1}^{\frac{n-1}{n-2}}\right\|_{L^{2^{*^{\prime}}\left(B_{\mu_{\alpha}}^{+}\right)}}\left(\mu_{\alpha}\left\|U_{1}^{\frac{n-1}{n-2}}\right\|_{L^{2^{*^{\prime}}}\left(B_{\mu_{\alpha}^{-1}}^{+}\right)}\right)\right. \\
\left.\left.+\epsilon_{\alpha}\left\|U_{1}^{r-1}\right\|_{L^{r}\left(\partial^{\prime} B_{\mu_{\alpha}^{-1}}^{+}\right)}+\mu_{\alpha}^{n-2}\left\|U_{1}^{q-2}\right\|_{L^{r}\left(\partial^{\prime} B_{\mu_{\alpha}^{-1}}^{+}\right)}\right)+\mu_{\alpha}^{2}\right\} .
\end{aligned}
$$


Due to $n \geq 5$, we have

$$
\begin{aligned}
\left\|U_{1}^{\frac{n-1}{n-2}}\right\|_{L^{2^{*^{\prime}}\left(B_{\mu_{\alpha}^{-1}}^{+}\right)}} & \leq C, \\
\mu_{\alpha}\left\|U_{1}^{r-1}\right\|_{L^{r}\left(\partial^{\prime} B_{\mu_{\alpha}^{-1}}^{+}\right)} & \leq C \mu_{\alpha}\left(1+\mu_{\alpha}^{\frac{n^{2}-8 n+8}{2 n}}\right)=o(1), \\
\left\|U_{1}^{q-2}\right\|_{L^{r}\left(\partial^{\prime} B_{\mu_{\alpha}^{-1}}^{+}\right)} & \leq C\left(1+\mu_{\alpha}^{(4-n) / 2}\right) .
\end{aligned}
$$

From (2.9), i.e., $\epsilon_{\alpha} \leq \alpha\left\|u_{\alpha}\right\|_{L^{r}(\partial M)}^{2}$, it follows that

$$
\alpha\left\|u_{\alpha}\right\|_{L^{r}(\partial M)}^{2} \leq C \mu_{\alpha}^{2} .
$$

On the other hand,

$$
\left\|u_{\alpha}\right\|_{L^{r}(\partial M)} \geq\left\|u_{\alpha}\right\|_{L^{r}\left(B_{\mu_{\alpha}}^{+}\left(x_{\alpha}\right) \cap \partial M\right)} \geq C^{-1} \mu_{\alpha}\left\|v_{\alpha}\right\|_{L^{r}\left(\partial^{\prime} B_{1}^{+}\right)} \geq C^{-1} \mu_{\alpha},
$$

where $v_{\alpha}$ is as in (2.7). Hence

$$
\alpha \leq C
$$

This is a contradiction.

3.1. Proof of Proposition 3.1. The rest of the paper is devoted to the proof of Proposition 3.1. We start with the equation that $w_{\alpha}$ satisfies.

Lemma 3.3. $w_{\alpha}$ satisfies

$$
\begin{cases}-\Delta_{\hat{g}} w_{\alpha}=t_{\alpha} \Delta_{\hat{g}} U_{\xi_{\alpha}, \lambda_{\alpha}}, & \text { in } B_{\delta_{\alpha}}^{+}, \\ \frac{\partial_{\hat{g} w}}{\partial \nu}-\ell_{\alpha}(q-1)\left|\Theta_{\alpha}\right|^{q-3} \Theta_{\alpha} w_{\alpha}+b^{\prime}\left|\Theta_{\alpha}\right|^{q-3} w_{\alpha}^{2}+b^{\prime \prime}\left|w_{\alpha}\right|^{q-1}=f_{\alpha}, & \text { on } \partial^{\prime} B_{\delta_{\alpha}}^{+},\end{cases}
$$

where

$$
\begin{aligned}
& \Theta_{\alpha}=t_{\alpha} \sigma_{\alpha}+\chi_{\alpha}, \\
& f_{\alpha}=t_{\alpha}\left(\ell_{\alpha} t_{\alpha}^{q-2}-\frac{1}{S}\right) U_{\xi_{\alpha}, \lambda_{\alpha}}^{q-1}-\alpha\left\|u_{\alpha}\right\|_{L^{r}(\partial M)}^{2-r} \psi_{\alpha}^{1-q} u_{\alpha}^{r-1}+O\left(\mu_{\alpha}^{(n-2) / 2}\right) U_{\xi_{\alpha}, \lambda_{\alpha}}^{q-2},
\end{aligned}
$$

and $b^{\prime}, b^{\prime \prime}$ are bounded functions with $b^{\prime} \equiv 0$ if $n \geq 4$.

Proof. The proof follows from straightforward computations. First of all, by the definition of $w_{\alpha}$ and by equation (3.1) of $u_{\alpha} / \psi_{\alpha}$, we have

$$
-\Delta_{\hat{g}} w_{\alpha}=-\Delta_{\hat{g}}\left(\frac{u_{\alpha}}{\psi_{\alpha}}-\chi_{\alpha}-t_{\alpha} \sigma_{\alpha}\right)=t_{\alpha} \Delta_{\hat{g}} \sigma_{\alpha}=t_{\alpha} \Delta_{\hat{g}} U_{\xi_{\alpha}, \lambda_{\alpha}} \quad \text { in } B_{\delta_{\alpha}}^{+},
$$

and

$$
\begin{aligned}
\frac{\partial_{\hat{g}} w_{\alpha}}{\partial \nu} & =\ell_{\alpha}\left(\Theta_{\alpha}+w_{\alpha}\right)^{q-1}-\alpha\left\|u_{\alpha}\right\|_{L^{r}(\partial M)}^{2-r} \psi_{\alpha}^{1-q} u_{\alpha}^{r-1}-t_{\alpha} \frac{\partial_{\hat{g}} \sigma_{\alpha}}{\partial \nu} \\
& =\ell_{\alpha}\left(\Theta_{\alpha}+w_{\alpha}\right)^{q-1}-\alpha\left\|u_{\alpha}\right\|_{L^{r}(\partial M)}^{2-r} \psi_{\alpha}^{1-q} u_{\alpha}^{r-1}-t_{\alpha} \frac{\partial_{\hat{g}} U_{\xi_{\alpha}, \lambda_{\alpha}}}{\partial \nu} \\
& =\ell_{\alpha}\left(\Theta_{\alpha}+w_{\alpha}\right)^{q-1}-\alpha\left\|u_{\alpha}\right\|_{L^{r}(\partial M)}^{2-r} \psi_{\alpha}^{1-q} u_{\alpha}^{r-1}-\frac{t_{\alpha}}{S} U_{\xi_{\alpha}, \lambda_{\alpha}}^{q-1}
\end{aligned}
$$

where we used that $\frac{\partial_{\hat{g}} U_{\xi_{\alpha}, \lambda_{\alpha}}}{\partial \nu}=\frac{\partial U_{\xi_{\alpha}, \lambda_{\alpha}}}{\partial x_{n}}=U_{\xi_{\alpha}, \lambda_{\alpha}}^{q-1}$ in Fermi coordinate systems. Note that we have the elementary expansion

$$
(k+l)^{q-1}=|k|^{q-2} k+(q-1)|k|^{q-3} k l+b^{\prime}(k, l)|k|^{q-3} l^{2}+b^{\prime \prime}(k, l)|l|^{q-1},
$$


for all $k, l$ such that $k+l>0$, where $b^{\prime}, b^{\prime \prime}$ are bounded and $b^{\prime} \equiv 0$ if $n \geq 4$. For $k=\Theta_{\alpha}, l=w_{\alpha}$, we obtain

$$
\begin{aligned}
\left(\Theta_{\alpha}+w_{\alpha}\right)^{q-1}= & \left|\Theta_{\alpha}\right|^{q-2} \Theta_{\alpha}+(q-1)\left|\Theta_{\alpha}\right|^{q-3} \Theta_{\alpha} w_{\alpha} \\
& +b^{\prime}\left|\Theta_{\alpha}\right|^{q-3} w_{\alpha}^{2}+b^{\prime \prime}\left|w_{\alpha}\right|^{q-1},
\end{aligned}
$$

and Lemma 3.3 follows from

$$
\begin{aligned}
\left|\Theta_{\alpha}\right|^{q-2} \Theta_{\alpha}= & \left|t_{\alpha} U_{\xi_{\alpha}, \lambda_{\alpha}}-t_{\alpha} h_{\alpha}+\chi_{\alpha}\right|^{q-2}\left(t_{\alpha} U_{\xi_{\alpha}, \lambda_{\alpha}}-t_{\alpha} h_{\alpha}+\chi_{\alpha}\right) \\
= & \left(t_{\alpha} U_{\xi_{\alpha}, \lambda_{\alpha}}\right)^{q-1}-\left(t_{\alpha} U_{\xi_{\alpha}, \lambda_{\alpha}}\right)^{q-2}\left(t_{\alpha} h_{\alpha}-\chi_{\alpha}\right) \\
& +\left|t_{\alpha} U_{\xi_{\alpha}, \lambda_{\alpha}}-\theta\left(t_{\alpha} h_{\alpha}+\chi_{\alpha}\right)\right|^{q-3}\left(t_{\alpha} h_{\alpha}+\chi_{\alpha}\right)\left(t_{\alpha} U_{\xi_{\alpha}, \lambda_{\alpha}}-t_{\alpha} h_{\alpha}+\chi_{\alpha}\right) \\
= & \left(t_{\alpha} U_{\xi_{\alpha}, \lambda_{\alpha}}\right)^{q-1}+O\left(\mu_{\alpha}^{(n-2) / 2} U_{\xi_{\alpha}, \lambda_{\alpha}}^{q-2}\right),
\end{aligned}
$$

where $\theta \in(0,1)$ and we have used 1) and 2) from Lemma 3.1 .

Define

$$
Q_{\alpha}(\varphi, \phi)=\int_{B_{\delta_{\alpha}}^{+}} \nabla_{\hat{g}} \varphi \nabla_{\hat{g}} \phi \mathrm{d} v_{\hat{g}}-\ell_{\alpha}(q-1) \int_{\partial^{\prime} B_{\delta_{\alpha}}^{+}}\left|\Theta_{\alpha}\right|^{q-3} \Theta_{\alpha} \varphi \phi \mathrm{d} s_{\hat{g}},
$$

for all $\varphi, \phi \in H_{0, L}\left(B_{\delta_{\alpha}}^{+}\right)$.

Lemma 3.4. There exist $0<\delta_{0}<<1, \alpha_{0} \geq 1$ and $c_{0}>0$ independent of $\alpha$ such that

$$
Q_{\alpha}(w, w) \geq c_{0} \int_{B_{\delta_{\alpha}}^{+}}\left|\nabla_{\hat{g}} w\right|^{2} \mathrm{~d} v_{\hat{g}}, \quad \forall w \in W_{\alpha}, \alpha \geq \alpha_{0} .
$$

Proof. The proof follows from Lemma 3.1 and Lemma 3.6

Proof of Proposition 3.1. Multiplying both sides of (3.8) by $w_{\alpha}$, we arrive at

$$
Q_{\alpha}\left(w_{\alpha}, w_{\alpha}\right)+o\left(\left\|w_{\alpha}\right\|^{2}\right)=t_{\alpha} \int_{B_{\delta_{\alpha}}^{+}} w_{\alpha} \Delta_{\hat{g}} U_{\xi_{\alpha}, \lambda_{\alpha}} \mathrm{d} v_{\hat{g}}+\int_{\partial^{\prime} B_{\delta_{\alpha}}^{+}} w_{\alpha} f_{\alpha} \mathrm{d} s_{\hat{g}},
$$

where we used

$$
\int_{\partial^{\prime} B_{\delta_{\alpha}}^{+}} b^{\prime \prime}\left|w_{\alpha}\right|^{q} \mathrm{~d} s_{\hat{g}} \leq C\left\|\nabla_{\hat{g}} w_{\alpha}\right\|_{L^{2}\left(B_{\delta_{\alpha}}^{+}\right)}^{q}=C\left\|w_{\alpha}\right\|^{2}\left\|w_{\alpha}\right\|^{q-2}
$$

and

$$
\int_{\partial^{\prime} B_{\delta_{\alpha}}^{+}} b^{\prime}\left|\Theta_{\alpha}\right|^{q-3}\left|w_{\alpha}\right|^{3} \leq C\left\|w_{\alpha}\right\|^{3}
$$

if $n=3$ and used also Lemma 3.1. By Hölder's inequality, Sobolev's inequality, and Lemma 3.4, we have

$$
\begin{aligned}
\left\|w_{a}\right\| \leq & C\left\{\left\|\Delta_{\hat{g}} U_{\xi_{\alpha}, \lambda_{\alpha}}\right\|_{L^{2^{*^{\prime}}\left(B_{\delta_{\alpha}}^{+}\right)}}+\alpha\left\|u_{\alpha}\right\|_{L^{r}(\partial M)}^{2-r}\left\|u_{\alpha}^{r-1}\right\|_{L^{q^{\prime}\left(\partial^{\prime} B_{\delta_{\alpha}}^{+}\right)}}\right. \\
& \left.+\mu_{\alpha}^{\frac{n-2}{2}}\left\|U_{\xi_{\alpha}, \lambda_{\alpha}}^{q-2}\right\|_{\left.L^{q^{\prime}\left(\partial^{\prime} B_{\delta_{\alpha}}^{+}\right)}\right\}}\right\}
\end{aligned}
$$


with $q^{\prime}=r=q /(q-1)$. Since $\Delta U_{\xi_{\alpha}, \lambda_{\alpha}}=0$, a direct computation in the Fermi coordinate system yields

$$
\begin{aligned}
& \left\|\Delta_{\hat{g}} U_{\xi_{\alpha}, \lambda_{\alpha}}\right\|_{L^{2^{*^{\prime}}}\left(B_{\delta_{\alpha}}^{+}\right)} \\
& =\left\|\Delta_{\hat{g}} U_{\xi_{\alpha}, \lambda_{\alpha}}-\Delta U_{\xi_{\alpha}, \lambda_{\alpha}}\right\|_{L^{2^{*^{\prime}}}\left(B_{\delta_{\alpha}}^{+}\right)} \\
& \leq\left\|\left(\hat{g}^{i j}-\delta^{i j}\right) \frac{\partial^{2} U_{\xi_{\alpha}, \lambda_{\alpha}}}{\partial z_{i} \partial z_{j}}-\hat{g}^{i j} \hat{\Gamma}_{i j}^{k} \frac{\partial u}{\partial z_{k}}\right\|_{L^{2^{*^{\prime}}}\left(B_{\delta_{\alpha}}^{+}\right)} \\
& \leq C\left(\int_{B_{\delta_{\alpha}}^{+}}\left(|z| \lambda_{\alpha}^{(n-2) / 2}\left(\left|z^{\prime}-\xi_{\alpha}^{\prime}\right|^{2}+\left(z_{n}+\lambda_{0} \lambda_{\alpha}\right)^{2}\right)^{-n / 2}\right)^{2^{*^{\prime}}} \mathrm{d} z\right)^{1 / 2^{*^{\prime}}} \\
& +C\left(\int_{B_{\delta_{\alpha}}^{+}}\left(\lambda_{\alpha}^{(n-2) / 2}\left(\left|z^{\prime}-\xi_{\alpha}^{\prime}\right|^{2}+\left(z_{n}+\lambda_{0} \lambda_{\alpha}\right)^{2}\right)^{-(n-1) / 2}\right)^{2^{*^{\prime}}} \mathrm{d} z\right)^{1 / 2^{*^{\prime}}} \\
& \leq C \lambda_{\alpha}\left(\int_{B_{\lambda_{\alpha}^{-1}}^{+}}\left(\left|y+\lambda_{\alpha}^{-1} \xi_{\alpha}\right|\left(\left|y^{\prime}\right|^{2}+\left(y_{n}+\lambda_{0}\right)^{2}\right)^{-n / 2}\right)^{2^{*^{\prime}}} \mathrm{d} y\right)^{1 / 2^{*^{\prime}}} \\
& +C \lambda_{\alpha}\left(\int_{B_{\lambda_{\alpha}^{-1}}}\left(\left(\left|y^{\prime}-\lambda_{\alpha}^{-1} \xi_{\alpha}\right|^{2}+\left(y_{n}+\lambda_{0}\right)^{2}\right)^{-(n-1) / 2}\right)^{2^{*^{\prime}}} \mathrm{d} y\right)^{1 / 2^{*^{\prime}}} \\
& \leq C \lambda_{\alpha}\left\|U_{1}^{\frac{n-1}{n-2}}\right\| \|_{L^{2^{*^{\prime}}}\left(B_{\lambda_{\alpha}^{-1}}^{+}\right)}
\end{aligned}
$$

where $2^{*}=\frac{2 n}{n-2}, 2^{*^{\prime}}=\frac{2 n}{n+2}$ and we used the variables transformation $y=$ $\lambda_{\alpha}^{-1}\left(z-\xi_{\alpha}\right)$ and the fact $\lambda_{\alpha}^{-1}\left|\xi_{\alpha}\right| \rightarrow 0$ as $\alpha \rightarrow \infty$. By Proposition 2.3, we have $u_{\alpha} \leq C U_{\mu_{\alpha}}$. Hence

$$
\left\|u_{\alpha}^{r-1}\right\|_{L^{q^{\prime}}\left(\partial^{\prime} B_{\delta_{\alpha}}^{+}\right)} \leq C\left\|U_{\mu_{\alpha}}^{r-1}\right\|_{L^{q^{\prime}\left(\partial^{\prime} B_{\delta_{\alpha}}^{+}\right)}} \leq \mu_{\alpha}^{n-1-\frac{n-2}{2} r}\left\|U_{1}^{r-1}\right\|_{L^{q^{\prime}}\left(\partial^{\prime} B_{\mu_{\alpha}^{-1}}^{+}\right)} .
$$

Finally, it is clear that

$$
\left\|U_{\xi_{\alpha}, \lambda_{\alpha}}^{q-2}\right\|_{L^{q^{\prime}}\left(\partial^{\prime} B_{\delta_{\alpha}}^{+}\right)} \leq C \mu_{\alpha}^{\frac{n-2}{2}}\left\|U_{1}^{q-2}\right\|_{L^{q^{\prime}}\left(\partial^{\prime} B_{\mu_{\alpha}^{-1}}^{+}\right.} .
$$

Therefore, we obtained the estimate for $\left\|w_{\alpha}\right\|$.

Multiplying (3.8) by $\sigma_{\alpha}$ and integrating over $B_{\delta_{\alpha}}^{+}$, we find that

$$
\begin{aligned}
\left|t_{\alpha}\left(\ell_{\alpha} t_{\alpha}^{q-2}-\frac{1}{S}\right)\right| & \int_{\partial^{\prime} B_{\delta_{\alpha}}^{+}} U_{\xi_{\alpha}, \lambda_{\alpha}}^{q-1} \sigma_{\alpha} \mathrm{d} s_{\hat{g}} \\
\leq & C\left(\int_{B_{\delta_{\alpha}}^{+}}\left|\Delta_{\hat{g}} U_{\xi_{\alpha}, \lambda_{\alpha}} \sigma_{\alpha}\right| \mathrm{d} v_{\hat{g}}+\int_{\partial^{\prime} B_{\delta_{\alpha}}^{+}} U_{\xi_{\alpha}, \lambda_{\alpha}}^{q-1}\left|w_{\alpha}\right| \mathrm{d} s_{\hat{g}}\right. \\
& \left.+\alpha\left\|u_{\alpha}\right\|_{L^{r}(\partial M)}^{2-r} \int_{\partial^{\prime} B_{\delta_{\alpha}}^{+}} U_{\xi_{\alpha}, \lambda_{\alpha}}^{r} \mathrm{~d} s_{\hat{g}}+\mu_{\alpha}^{(n-2) / 2} \int_{\partial^{\prime} B_{\delta_{\alpha}}^{+}} U_{\xi_{\alpha}, \lambda_{\alpha}}^{q-1} \mathrm{~d} s_{\hat{g}}\right),
\end{aligned}
$$

where we used $\left|\sigma_{\alpha}\right|+\left|\Theta_{\alpha}\right|+\left|w_{\alpha}\right|+u_{\alpha} \leq C U_{\xi_{\alpha}, \lambda_{\alpha}}$. Since $\int_{\partial^{\prime} B_{\delta_{\alpha}}^{+}} U_{\xi_{\alpha}, \lambda_{\alpha}}^{q-1} \sigma_{\alpha} \mathrm{d} s_{\hat{g}} \geq$ $C^{-1}>0$, the estimate of $\left|\ell_{\alpha} t_{\alpha}^{q-2}-\frac{1}{S}\right|$ follows by Hölder's inequality and the estimate for $\left\|w_{\alpha}\right\|$. 
3.2. Proof of Lemma 3.4. Let $\mathcal{D}^{1,2}\left(\mathbb{R}_{+}^{n}\right)$ be the closure of $C_{c}^{\infty}\left(\mathbb{R}_{+}^{n} \cup \partial \mathbb{R}_{+}^{n}\right)$ under the norm

$$
\|u\|_{\mathcal{D}^{1,2}\left(\mathbb{R}_{+}^{n}\right)}=\left(\int_{\mathbb{R}_{+}^{n}}|\nabla u|^{2} \mathrm{~d} y\right)^{1 / 2} .
$$

In fact, $\mathcal{D}^{1,2}\left(\mathbb{R}_{+}^{n}\right)$ is a Hilbert space with the inner product

$$
\langle\varphi, \phi\rangle_{1}:=\int_{\mathbb{R}_{+}^{n}} \nabla \varphi \nabla \phi \mathrm{d} y
$$

for any $\varphi, \phi \in \mathcal{D}^{1,2}\left(\mathbb{R}_{+}^{n}\right)$. Define the functional

$$
Q_{1}(\varphi, \phi):=\int_{\mathbb{R}_{+}^{n}} \nabla \varphi \nabla \phi \mathrm{d} y-\frac{q-1}{S} \int_{\partial \mathbb{R}_{+}^{n}} U_{1}^{q-2} \varphi \phi \mathrm{d} y^{\prime}
$$

for all $\varphi, \phi \in E_{0}$, where $y=\left(y^{\prime}, 0\right)$ and

$$
\begin{gathered}
E_{1}=\left\{w \in \mathcal{D}^{1,2}\left(\mathbb{R}_{+}^{n}\right): \quad\left\langle\left.\frac{\partial U_{\xi, 1}}{\partial \xi_{i}}\right|_{\xi=0}, w\right\rangle_{1}=\left\langle\left.\frac{\partial U_{0, \lambda}}{\partial \lambda}\right|_{\lambda=1}, w\right\rangle_{1}=\left\langle U_{0,1}, w\right\rangle_{1}=0\right. \\
i=1,2, \cdots, n-1\} .
\end{gathered}
$$

Then we have

Lemma 3.5. There exists a constant $c_{1}>0$ depending only on $n$ such that

$$
Q_{1}(w, w) \geq c_{1}\|w\|_{\mathcal{D}^{1,2}\left(\mathbb{R}_{+}^{n}\right)}^{2},
$$

for all $w \in E_{1}$

Lemma 3.5 follows from an analysis of the eigenvalues of

$$
\begin{cases}\Delta v=0, & \text { in } B_{1} \\ \frac{\partial v}{\partial \nu}=\lambda v, & \text { on } \partial B_{1} .\end{cases}
$$

The details can be found in, e.g., 9].

Lemma 3.6. For $R>0$ and $x \in \partial \mathbb{R}^{n}$ with $|x| \leq R / 10$, let $h_{i j}$ be a Riemannian metric on $B_{R}^{+}(x), k>0$ and $\Theta \in L^{q}\left(\partial^{\prime} B_{R}\right)$. Denote $Q_{2}$ as the continuous bilinear form on $H_{0, L}\left(B_{R}^{+}(x)\right) \times H_{0, L}\left(B_{R}^{+}(x)\right)$

$$
Q_{2}(\varphi, \phi)=\int_{B_{R}^{+}(x)} \nabla_{h} \varphi \nabla_{h} \phi \mathrm{d} v_{h}-k \int_{\partial^{\prime} B_{R}^{+}(x)}|\Theta|^{q-3} \Theta \varphi \phi, \mathrm{d} s_{h} .
$$

There exists a small positive $\varepsilon_{0}$ depending only on $n$ such that if

$$
\left\|\Theta-U_{1}\right\|_{L^{q}\left(\partial^{\prime} B_{R}^{+}(x)\right)}+\left|k-\frac{q-1}{S}\right|+\left\|h_{i j}-\delta_{i j}\right\|_{L^{\infty}\left(B_{R}^{+}(x)\right)} \leq \varepsilon_{0},
$$

then

for all $w \in E_{2}$, where

$$
Q_{2}(w, w) \geq \frac{c_{1}}{2} \int_{B_{R}^{+}(x)}\left|\nabla_{h} w\right|^{2} \mathrm{~d} v_{h}
$$

$$
\begin{aligned}
E_{2}=\left\{w \in H_{0, L}\left(B_{R}^{+}(x)\right):\right. & \left\langle U_{0,1}, w\right\rangle_{1} \leq \varepsilon_{0}\|w\|_{h},\left\langle\left.\frac{\partial U_{0, \lambda}}{\partial \lambda}\right|_{\lambda=1}, w\right\rangle_{1} \leq \varepsilon_{0}\|w\|_{h} \\
& \left.\left\langle\left.\frac{\partial U_{\xi, 1}}{\partial \xi_{i}}\right|_{\xi=0}, w\right\rangle_{1} \leq \varepsilon_{0}\|w\|_{h}, i=1,2, \cdots, n-1\right\} .
\end{aligned}
$$


Proof. From the assumptions, there exist $\delta_{1}, \cdots, \delta_{n+1}$ satisfying $\left|\delta_{j}\right|=O\left(\varepsilon_{0}\|w\|_{h}\right)$ for every $j$ such that

$$
\tilde{w}=w-\left.\sum_{j=1}^{n-1} \delta_{j} \frac{\partial U_{\xi, 1}}{\partial \xi_{i}}\right|_{\xi=0}-\left.\delta_{n} \frac{\partial U_{0, \lambda}}{\partial \lambda}\right|_{\lambda=1}-\delta_{n+1} U_{0,1}
$$

belongs to $E_{1}$. It follows from Lemma 3.5 that

$$
Q_{1}(\tilde{w}, \tilde{w}) \geq c_{1}\|\tilde{w}\|_{\mathcal{D}^{1,2}\left(\mathbb{R}_{+}^{n}\right)} .
$$

The lemma follows by choosing sufficiently small $\varepsilon_{0}$ and making use of the Sobolev trace inequality.

\section{ACKNOWLEDGEMENTS}

Both authors would like to thank Professor YanYan Li for his interest in the work and constant encouragement.

\section{REFERENCES}

[1] Adimurthi and S. L. Yadava, Some remarks on Sobolev type inequalities, Calc. Var. Partial Differential Equations 2 (1994), no. 4, 427-442, DOI 10.1007/BF01192092. MR1383917 (97e:35033)

[2] Thierry Aubin, Problèmes isopérimétriques et espaces de Sobolev (French), J. Differential Geometry 11 (1976), no. 4, 573-598. MR0448404 (56 \#6711)

[3] Thierry Aubin and Yan Yan Li, On the best Sobolev inequality, J. Math. Pures Appl. (9) 78 (1999), no. 4, 353-387, DOI 10.1016/S0021-7824(99)00012-4. MR1696357 (2000e:46041)

[4] A. Bahri and J.-M. Coron, The scalar-curvature problem on the standard three-dimensional sphere, J. Funct. Anal. 95 (1991), no. 1, 106-172, DOI 10.1016/0022-1236(91)90026-2. MR.1087949 (92k:58055)

[5] William Beckner, Sharp Sobolev inequalities on the sphere and the Moser-Trudinger inequality, Ann. of Math. (2) 138 (1993), no. 1, 213-242, DOI 10.2307/2946638. MR1230930 (94m:58232)

[6] Haïm Brezis and Elliott H. Lieb, Sobolev inequalities with remainder terms, J. Funct. Anal. 62 (1985), no. 1, 73-86, DOI 10.1016/0022-1236(85)90020-5. MR790771 (86i:46033)

[7] Haïm Brézis and Walter A. Strauss, Semi-linear second-order elliptic equations in $L^{1}$, J. Math. Soc. Japan 25 (1973), 565-590. MR0336050 (49 \#826)

[8] Pascal Cherrier, Problèmes de Neumann non linéaires sur les variétés riemanniennes (French, with English summary), J. Funct. Anal. 57 (1984), no. 2, 154-206, DOI 10.1016/0022-1236(84)90094-6. MR749522 (86c:58154)

[9] Sérgio de Moura Almaraz, Blow-up phenomena for scalar-flat metrics on manifolds with boundary, J. Differential Equations 251 (2011), no. 7, 1813-1840, DOI 10.1016/j.jde.2011.04.013. MR2823676

[10] Olivier Druet, The best constants problem in Sobolev inequalities, Math. Ann. 314 (1999), no. 2, 327-346, DOI 10.1007/s002080050297. MR.1697448(2000d:58033)

[11] Olivier Druet, Isoperimetric inequalities on compact manifolds, Geom. Dedicata 90 (2002), 217-236, DOI 10.1023/A:1014977309741. MR1898162(2003a:53045)

[12] Olivier Druet and Emmanuel Hebey, The AB program in geometric analysis: sharp Sobolev inequalities and related problems, Mem. Amer. Math. Soc. 160 (2002), no. 761, viii+98, DOI 10.1090/memo/0761. MR:1938183 (2003m:58028)

[13] José F. Escobar, Conformal deformation of a Riemannian metric to a scalar flat metric with constant mean curvature on the boundary, Ann. of Math. (2) 136 (1992), no. 1, 1-50, DOI 10.2307/2946545. MR:1173925 (93e:53046)

[14] José F. Escobar, Sharp constant in a Sobolev trace inequality, Indiana Univ. Math. J. 37 (1988), no. 3, 687-698, DOI 10.1512/iumj.1988.37.37033. MR962929(90a:46071)

[15] N. Ghoussoub and F. Robert, The effect of curvature on the best constant in the HardySobolev inequalities, Geom. Funct. Anal. 16 (2006), no. 6, 1201-1245, DOI 10.1007/s00039006-0579-2. MR2276538(2007k:35085) 
[16] Fengbo Hang, Xiaodong Wang, and Xiaodong Yan, An integral equation in conformal geometry, Ann. Inst. H. Poincaré Anal. Non Linéaire 26 (2009), no. 1, 1-21, DOI 10.1016/j.anihpc.2007.03.006. MR2483810 (2010a:53050)

[17] Emmanuel Hebey, Nonlinear analysis on manifolds: Sobolev spaces and inequalities, Courant Lecture Notes in Mathematics, vol. 5, New York University, Courant Institute of Mathematical Sciences, New York; American Mathematical Society, Providence, RI, 1999. MR.1688256 (2000e:58011)

[18] Emmanuel Hebey, Sharp Sobolev-Poincaré inequalities on compact Riemannian manifolds, Trans. Amer. Math. Soc. 354 (2002), no. 3, 1193-1213 (electronic), DOI 10.1090/S0002-994701-02913-0. MR 1867378 (2002m:58025)

[19] Emmanuel Hebey and Michel Vaugon, Meilleures constantes dans le théorème d'inclusion de Sobolev (French, with English and French summaries), Ann. Inst. H. Poincaré Anal. Non Linéaire 13 (1996), no. 1, 57-93. MR1373472 (96m:46054)

[20] Tianling Jin and Jingang Xiong, A fractional Yamabe flow and some applications, to appear in J. Reine Angew. Math. DOI:10.1515/crelle-2012-0110.

[21] Tianling Jin and Jingang Xiong, Sharp constants in weighted trace inequalities on Riemannian manifolds, Calc. Var. Partial Differential Equations 48 (2013), no. 3-4, 555-585, DOI 10.1007/s00526-012-0562-8. MR 3116023

[22] Carlos E. Kenig and Jill Pipher, The Neumann problem for elliptic equations with nonsmooth coefficients, Invent. Math. 113 (1993), no. 3, 447-509, DOI 10.1007/BF01244315. MR.1231834 (95b:35046)

[23] Yanyan Li and Tonia Ricciardi, A sharp Sobolev inequality on Riemannian manifolds, Commun. Pure Appl. Anal. 2 (2003), no. 1, 1-31, DOI 10.1016/S1631-073X(02)02529-3. MR.1961446(2004a:58039)

[24] Yanyan Li and Meijun Zhu, Uniqueness theorems through the method of moving spheres, Duke Math. J. 80 (1995), no. 2, 383-417, DOI 10.1215/S0012-7094-95-08016-8. MR1369398 (96k:35061)

[25] Yanyan Li and Meijun Zhu, Sharp Sobolev trace inequalities on Riemannian manifolds with boundaries, Comm. Pure Appl. Math. 50 (1997), no. 5, 449-487, DOI 10.1002/(SICI)10970312(199705)50:5〈449::AID-CPA2〉3.3.CO;2-5. MR.1443055 (98c:46065)

[26] Yanyan Li and Meijun Zhu, Sharp Sobolev inequalities involving boundary terms, Geom. Funct. Anal. 8 (1998), no. 1, 59-87, DOI 10.1007/s000390050048. MR1601846 (98m:58144)

[27] Elliott H. Lieb, Sharp constants in the Hardy-Littlewood-Sobolev and related inequalities, Ann. of Math. (2) 118 (1983), no. 2, 349-374, DOI 10.2307/2007032. MR717827(86i:42010)

Department of Mathematics, The University of Chicago, 5734 S. University Avenue, Chichgo, Illinois 60637

E-mail address: tj@math.uchicago.edu

Beijing International Center for Mathematical Research, Peking University, BeiJING 100871, CHINA

E-mail address: jxiong@math.pku.edu.cn 